\title{
Computing of Fluidic Forces in Rotating Cylinders with Application to Active Magnetic Bearing Control
}

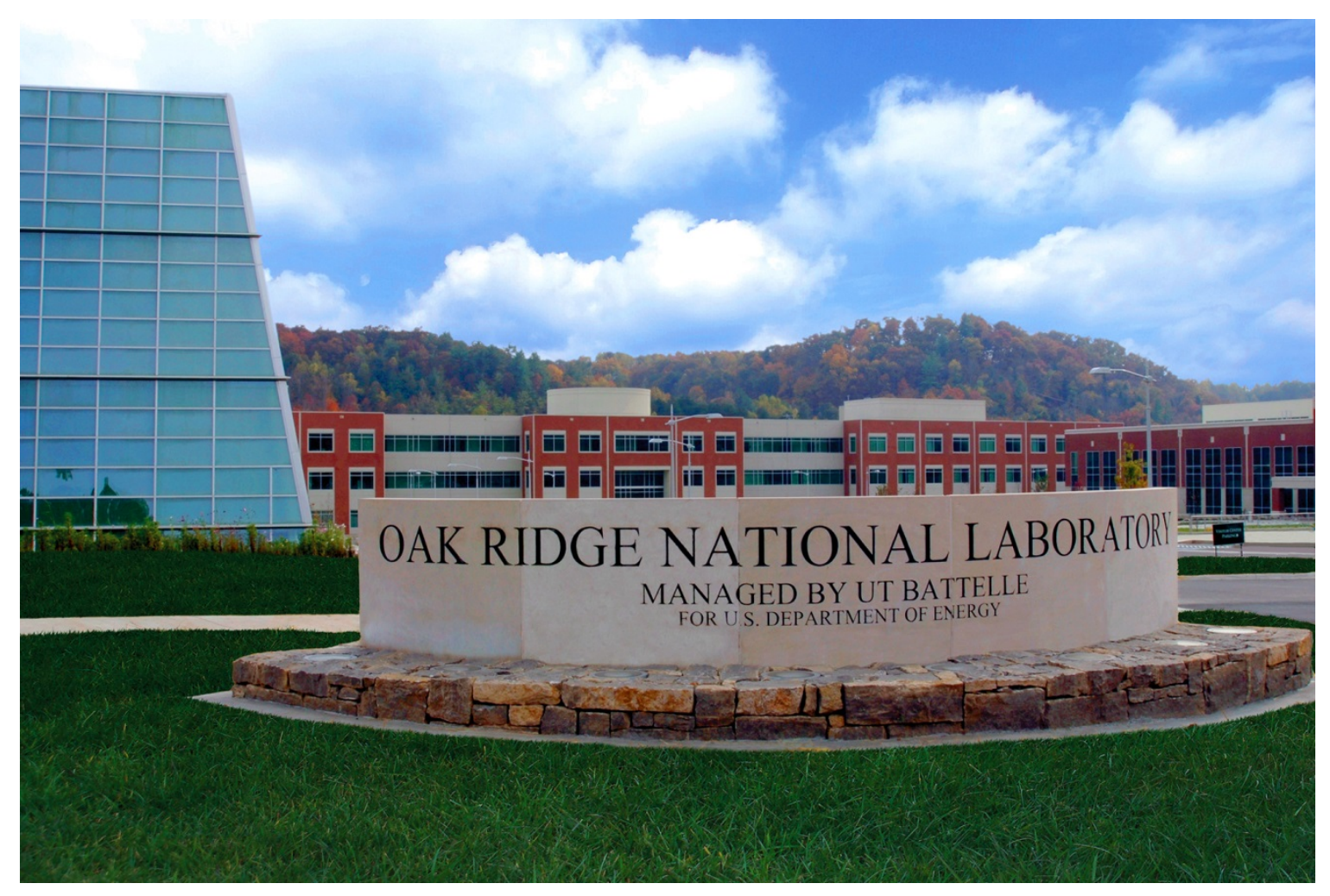

Emilian Popov

November 2021 


\title{
DOCUMENT AVAILABILITY
}

Reports produced after January 1, 1996, are generally available free via US Department of Energy (DOE) SciTech Connect.

Website www.osti.gov

Reports produced before January 1, 1996, may be purchased by members of the public from the following source:

\author{
National Technical Information Service \\ 5285 Port Royal Road \\ Springfield, VA 22161 \\ Telephone 703-605-6000 (1-800-553-6847) \\ TDD 703-487-4639 \\ Fax 703-605-6900 \\ E-mail info@ntis.gov \\ Website http://classic.ntis.gov/
}

Reports are available to DOE employees, DOE contractors, Energy Technology Data Exchange representatives, and International Nuclear Information System representatives from the following source:

Office of Scientific and Technical Information

PO Box 62

Oak Ridge, TN 37831

Telephone 865-576-8401

Fax 865-576-5728

E-mail reports@osti.gov

Website https://www.osti.gov/

This report was prepared as an account of work sponsored by an agency of the United States Government. Neither the United States Government nor any agency thereof, nor any of their employees, makes any warranty, express or implied, or assumes any legal liability or responsibility for the accuracy, completeness, or usefulness of any information, apparatus, product, or process disclosed, or represents that its use would not infringe privately owned rights. Reference herein to any specific commercial product, process, or service by trade name, trademark, manufacturer, or otherwise, does not necessarily constitute or imply its endorsement, recommendation, or favoring by the United States Government or any agency thereof. The views and opinions of authors expressed herein do not necessarily state or reflect those of the United States Government or any agency thereof. 
Nuclear Energy and Fuel Cycle Division

\title{
COMPUTING OF FLUIDIC FORCES IN ROTATING CYLINDERS WITH APPLICATION TO ACTIVE MAGNETIC BEARING CONTROL
}

\author{
Emilian Popov
}

November 2021

\author{
Prepared by \\ OAK RIDGE NATIONAL LABORATORY \\ Oak Ridge, TN 37831-6283 \\ managed by \\ UT-BATTELLE LLC \\ for the \\ US DEPARTMENT OF ENERGY \\ under contract DE-AC05-00OR22725
}




\section{CONTENTS}

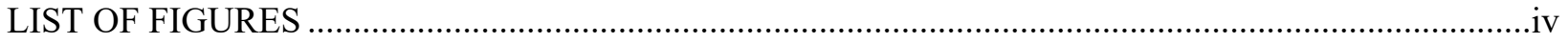

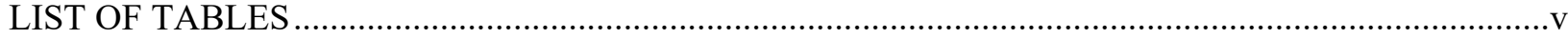

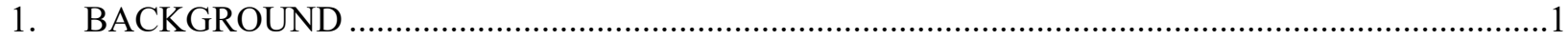

2. COUETTE FLOWS: TRANSITION TO VORTICIAL FLOW STRUCTURE, CRITICAL RE

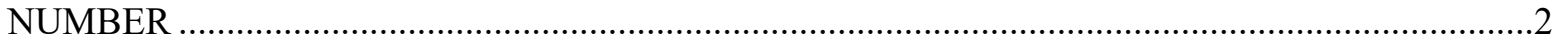

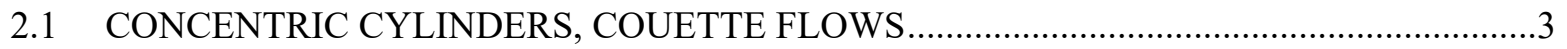

2.2 ECCENTRIC CYLINDERS IN STABLE COUETTE FLOWS ............................................

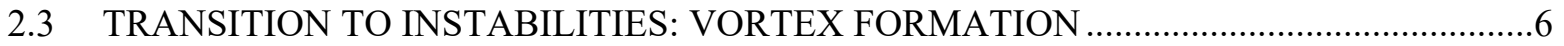

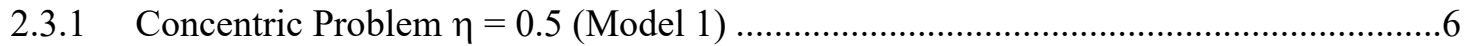

2.3.2 Eccentric Problem $\eta=0.5, \varepsilon=0.67$ (Model 1) ...........................................................

2.3.3 Concentric Problem $\eta=0.98$ (Model 2) ..................................................................

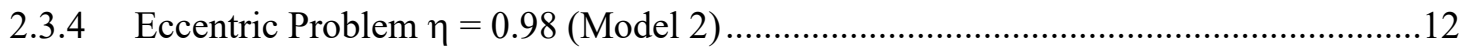

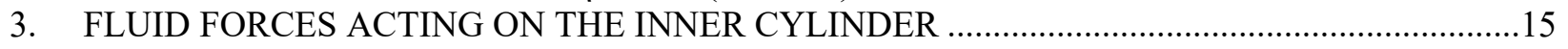

4. COMPUTING OF FORCES IN HIGH-SPEED ROTATIONAL FLOWS: CONCENTRIC

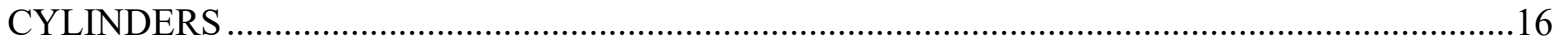

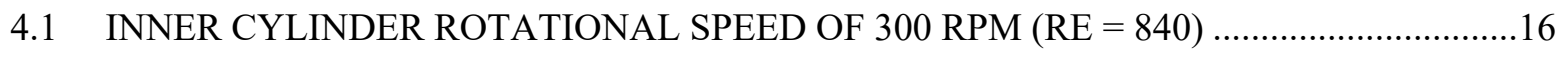

4.2 ROTATIONAL SPEEDS OF 600 RPM, OR $10 \mathrm{HZ}(\mathrm{RE}=1,680)$ : EFFECT OF THE

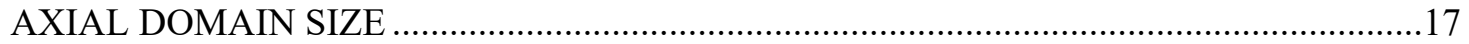

4.3 ROTATIONAL SPEEDS CLOSE TO RATED $(1,200-2,400$ RPM) .................................... 19

4.4 SUMMARY OF CONCLUSIONS FOR THE CONCENTRIC PROBLEM............................22

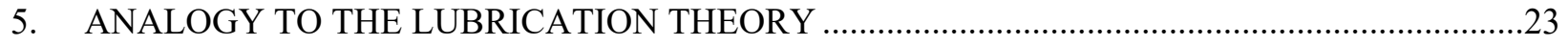

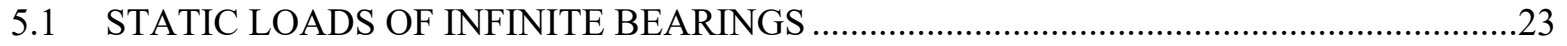

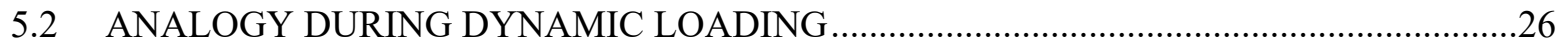

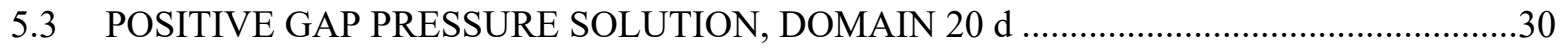

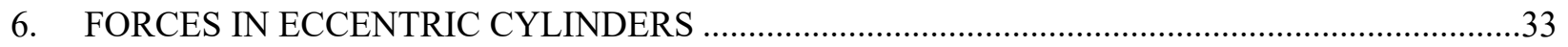

6.1 LINEAR ROTOR MOTION, LONG BEARING ANALOG ................................................33

6.2 SINE MOTION OF THE INNER CYLINDER, POSITIVE PRESSURE ANALOG,

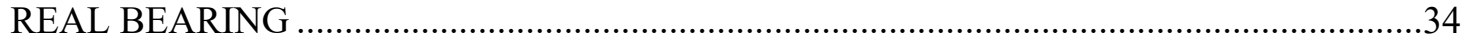

6.3 ROTATIONAL SPEED IMPACT ON PHASE SHIFT AND FORCE MAGNITUDE ............35

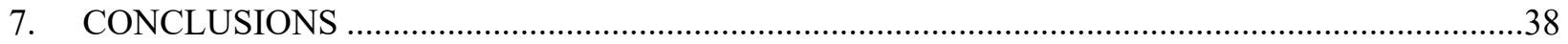




\section{LIST OF FIGURES}

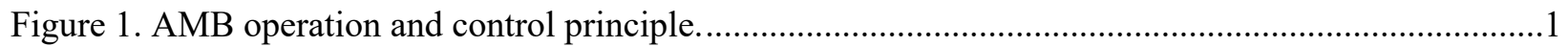

Figure 2. Computational models, geometries, and grids: Ri/d = 1 (left), Ri/d = 44 (right)........................

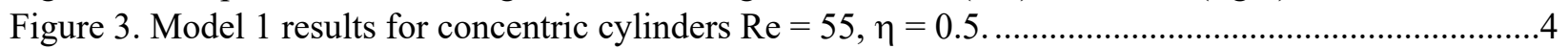

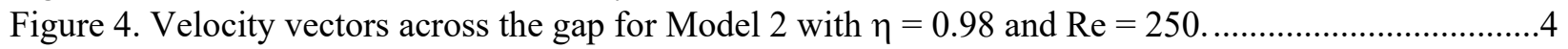

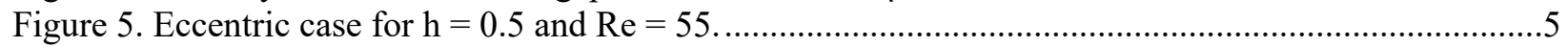

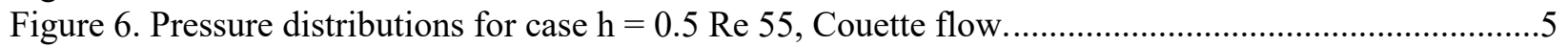

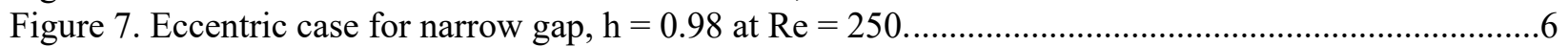

Figure 8. Schematic of counter-rotating axisymmetric vortices of Taylor-Couette flow (R.

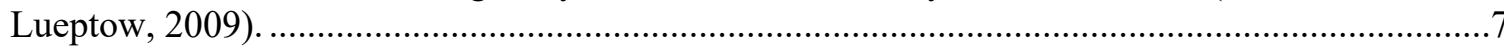

Figure 9. Vortex formation for the eccentric Model 1 problem: velocity (left) and vorticity (right)

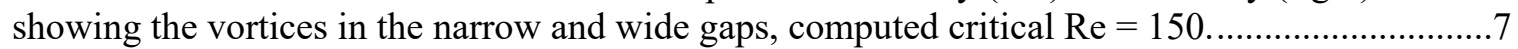

Figure 10. Static pressures for $\mathrm{Re}=55$ stable (left), and $\mathrm{Re}=150$ instability inception (right)...............8

Figure 11. Model 2 result at $R e=400$, converged transient simulation with a timestep of 0.001 after $3 \mathrm{~s}$ (7 revolutions).

Figure 12. Couette flow regime for narrow gap $\eta=0.99$ (D. Deng, 2007) (left), and $\eta=0.98$ from

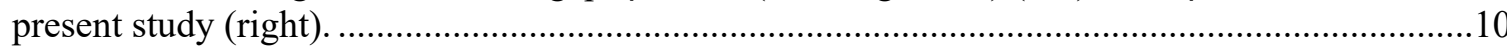

Figure 13. Taylor vortex flow regime for narrow gap $\eta=0.99$ (D. Deng, 2007) (left), and $\eta=0.98$ from present study (right).

Figure 14. Wavy vortex flow regime for narrow gap $\eta=0.99$ (Deng, 2007) (left), and $\eta=0.98$ from present study (right).

Figure 15. Eccentric case, narrow gap $(\eta=0.98)$ beginning of transition to vortex wavy flow at $\mathrm{Re}=550$.

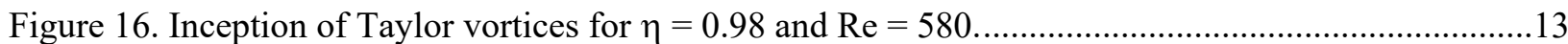

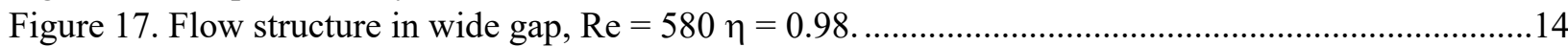

Figure 18. Forces on the inner cylinder for a speed of $300 \mathrm{rpm}$ and an axial domain of $6 \mathrm{~d}$. ....................16

Figure 19. Pressure on the inner wall (left) and the radial velocity in the gap (right) for a speed of $300 \mathrm{rpm}$, domain $6 \mathrm{~d}$.

Figure 20. $X$ component of vortex-induced force for three axial domain sizes $-3,6$, and $9 \mathrm{~d}-$ plotted on the same scale for comparison.

Figure 21. Vortex pattern as pressure imprint on the inner (rotating) cylinder wall (left) and particle tracks for one revolution (right) for the three domains - 3, 6, and $9 \mathrm{~d}$ (from bottom up).....

Figure 22. Combined plots of force, pressure imprint of the inner cylinder, particle track, and transverse gap velocity (vortex pattern) for rotational speeds of 1,200 (top), 1,800 (middle) and 2,400 (bottom) rpm.

Figure 23. Combined plots from $3 \mathrm{~d}$ model calculations at rotational speeds of 1,200 and 1,500 rpm.

Figure 24. The plot on the left shows the Sommerfield (S) number calculation for the pump rotor and a comparison to theoretical solution for infinitely long bearings (left), accounting for the negative pressure (green line), and positive pressure (red markers) (O. Pinkus, 1961), .........25

Figure 25. Static loading pressures from numerical solution (red), long bearing (blue), and short

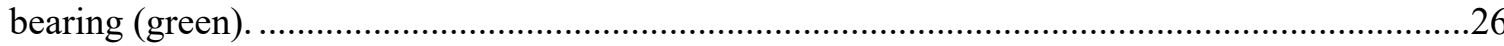

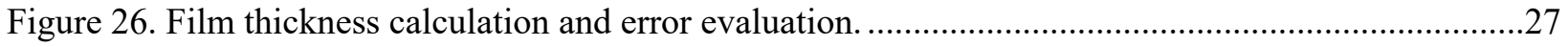

Figure 27. Forces during dynamic (25-25.01 s) and static (25.01-25.02) loading for $1500 \mathrm{rpm} . \ldots \ldots \ldots \ldots . . .29$

Figure 28. Loading pressures at the end of dynamic linear motion with $6 \mathrm{~cm} / \mathrm{s}$ from numerical the solution (red), long bearing (blue), and short bearing (green). 
Figure 29. Midline gap pressure at the end of the linear motion (top), and during the static operation (bottom), for the three configurations: simulation (red), long bearing (blue), and short bearing (green).

Figure 30. Forces during dynamic and static loading with constant pressure boundary conditions and finite rotor length ( $20 \mathrm{~d}$ ) for simulation (solid line) and lubrication theory (dotted lines).

Figure 31. Gap pressure distribution in the simulation in the static regime in the vicinity of minimal gap.

Figure 32. Dynamic and static forces during and after a linear motion of rotor (journal) of $6 \mathrm{~cm} / \mathrm{s}$ and a maximum relative eccentricity $\varepsilon=0.8$.

Figure 33. Forces (left) and rotor position (right) during sine motion with a frequency of 31.83 $\mathrm{Hz}$; two cycles modeled.

Figure 34. Pressures during sine motion. The pressure imprint on the inner cylinder wall is shown on the left, and the midpoint pressure distribution along the circumference for simulation pressure (red), long bearing (blue), and short bearing (green) are plotted on the right. 35

Figure 35. Phase shift evaluation plot with normalized $X$ and $Y$ forces from simulation.

Figure 36. Rotational speed effect. $X$ (left) and $Y$ (right) forces resulting from $31.8 \mathrm{~Hz}$ sine motion are plotted for three rotational speeds: $600,1,200$, and $1,800 \mathrm{rpm}$

\section{LIST OF TABLES}

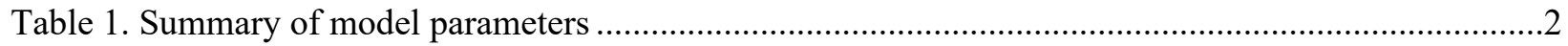

Table 2. Instabilities resulting from rotation of inner cylinder ..............................................................6

Table 3. Summary of meshing sensitivity to modeling the transition to vortices for $\eta=0.98 \ldots \ldots \ldots \ldots \ldots \ldots . . .9$

Table 4. Data for comparative calculations used to validate results for Taylor and wavy flow regimes

Table 5. Simulation input data for linear motion of inner cylinder in the $X$ direction with a maximum displacement of $0.6 \mathrm{~mm}$ 


\section{BACKGROUND}

Oak Ridge National Laboratory (ORNL) has been engaged to develop molten salt technologies. Molten salts are chemically aggressive liquids that operate at high temperatures. As part of this activity, ORNL designed a molten salt pump for molten salt that has no seals and that operates immersed in the salt. To minimize wear on rotating components, the pump uses active magnetic bearings (AMBs) to support the shaft and rotor. The pump is centrifugal and is oriented horizontally (A. Melin, 2013).

The AMB system requires active control to operate. Figure 1 shows the AMB principle of operation and control (Calnetics, 2020). A ferrous object (pump rotor) levitates in a magnetic field created by an electromagnet. The force of gravity is balanced by the electromagnetic force.

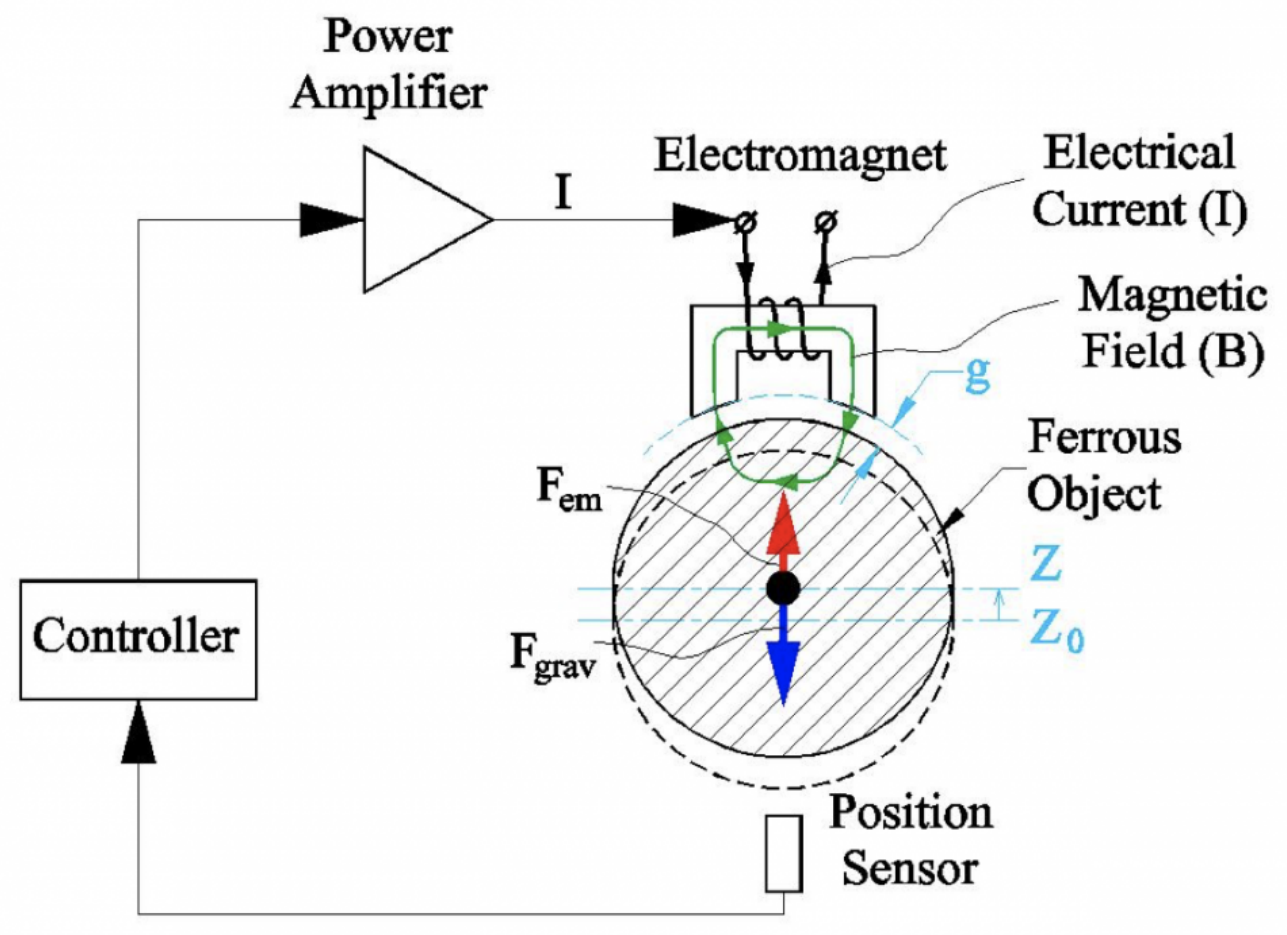

Figure 1. AMB operation and control principle.

As the rotor deviates from its initial position, there is no inherent self-controlled mechanism to return the rotor back to its original position. Therefore, the magnetic field is used for adjustment. Typically, a position sensor detects the deviation, and a controller is used to adjust the current to the electromagnet.

The specific AMB control as applied to an immersed pump rotor relies on the mechanical forces acting on the rotor, as well as the forces from the rotating fluid. Melin et al. (A. Melin, 2013) developed a rotor mechanical dynamic model to aid the AMB control. The model employs two matrices of coefficients to account for the fluidic effects: a damping matrix, and a stiffness matrix. The goal of the computational fluid dynamics (CFD) modeling and simulation was to help define those coefficients. The STAR-CCM+ commercial CFD package was used (Siemens, 2020). 


\section{COUETTE FLOWS: TRANSITION TO VORTICIAL FLOW STRUCTURE, CRITICAL RE NUMBER}

The pump assembly has a rotor with a shaft connected to the impeller, and a stator contains the electromagnets. The fluid (salt) fills in the assembly, thus eliminating the need for seals. This type of pump is known as a canned pump.

The fluid problem was approximated with two concentric cylinders (rotor and stator), and only the inner cylinder rotates. The fluid in the gap between the rotor and the stator is subjected to shear rotational forces. At low speeds, the exact solution of the Navier-Stokes equations for an incompressible flow in the gap between the two cylinders results in only the radial velocity component, or Couette flow (M. Couette, 1890). Two types of force balance to stabilize the flow: a centrifugal force caused by the rotating inner cylinder, and a pressure gradient force from the velocity distribution in the gap. Recktenwald (A. Recktenwald, 1993) defined a velocity-based Reynolds number (Re) at the inner cylinder surface that can be used to characterize the flow in the gap (d):

$$
\operatorname{Re}=\Omega \mathrm{R}_{\mathrm{i}} \mathrm{d} / \mathrm{v}
$$

where

$\Omega$ is rotational speed, $\mathrm{rad} / \mathrm{s}$;

$\mathrm{R}_{\mathrm{i}}$ is the inner cylinder's radius, $\mathrm{m}$;

$\mathrm{d}=\left(\mathrm{R}_{\mathrm{o}}-\mathrm{R}_{\mathrm{i}}\right)-$ gap, $\mathrm{m}$; and

$v$ is kinematic viscosity, $\mathrm{m}^{2} / \mathrm{s}$.

To analyze the Couette flow and verify the numerical solution, two computational models were developed with small and large rotor radius-to-gap ratios. Using the gap (d) as a length scale, the model dimensions were as follows:

Model 1: $R_{i}=d, R_{o}=2 d,(\eta=0.5)$, length $L=3 d$, Model 2 (closer to the real pump model): $\mathrm{Ri}=44 \mathrm{~d}, \mathrm{R}_{\mathrm{o}}=45 \mathrm{~d}(\eta=44 / 45=0.98)$, length $\mathrm{L}=3 \mathrm{~d}$.

The models assume axial periodicity equivalent to cylinders of infinite length. The verification aimed to show that the viscosity forces that damp the instabilities at low speeds are properly calculated. It is also helpful to define requirements to the spatial discretization and modeling methods. Table 1 summarizes some of the key model parameters.

Table 1. Summary of model parameters

\begin{tabular}{|l|l|l|l|}
\hline \multirow{4}{*}{ Computational grid parameters } & \multicolumn{2}{|l|}{ Hexahedral elements } \\
\cline { 2 - 3 } & Double-sided 6-element boundary layers \\
\cline { 2 - 3 } & & Model 1 & Model 2 \\
\cline { 2 - 3 } & Gap resolution & 50 & $20-30$ \\
\hline \multirow{5}{*}{ Solution models } & Total grid elements & $4.6 \mathrm{M}$ & $3-59 \mathrm{M}$ \\
\hline & Segregated steady solution & \\
\hline & Viscous laminar flow \\
\hline & Second-order convection flux \\
\cline { 2 - 3 } Boundary conditions & Constant density \\
\hline & Axial periodicity \\
\cline { 2 - 3 } & Prescribed inner wall rotational velocity \\
\hline
\end{tabular}


If the gap resolution is kept the same, then the large $R_{i}-t o-R_{o}(\eta)$ ratio leads to an increased number of elements. The gap resolution for Model 2 was varied between 20 and 40, which produced grids with elements between 3 and 59 million (Table 1), depending on the meshing algorithm. Another possible reason for reducing the grid size is to model the geometry in partial symmetry or in frames of reference. This option was not explored because of uncertainties with the modeling of the offset configuration. The two model geometries and computational grids are shown in Figure 2.

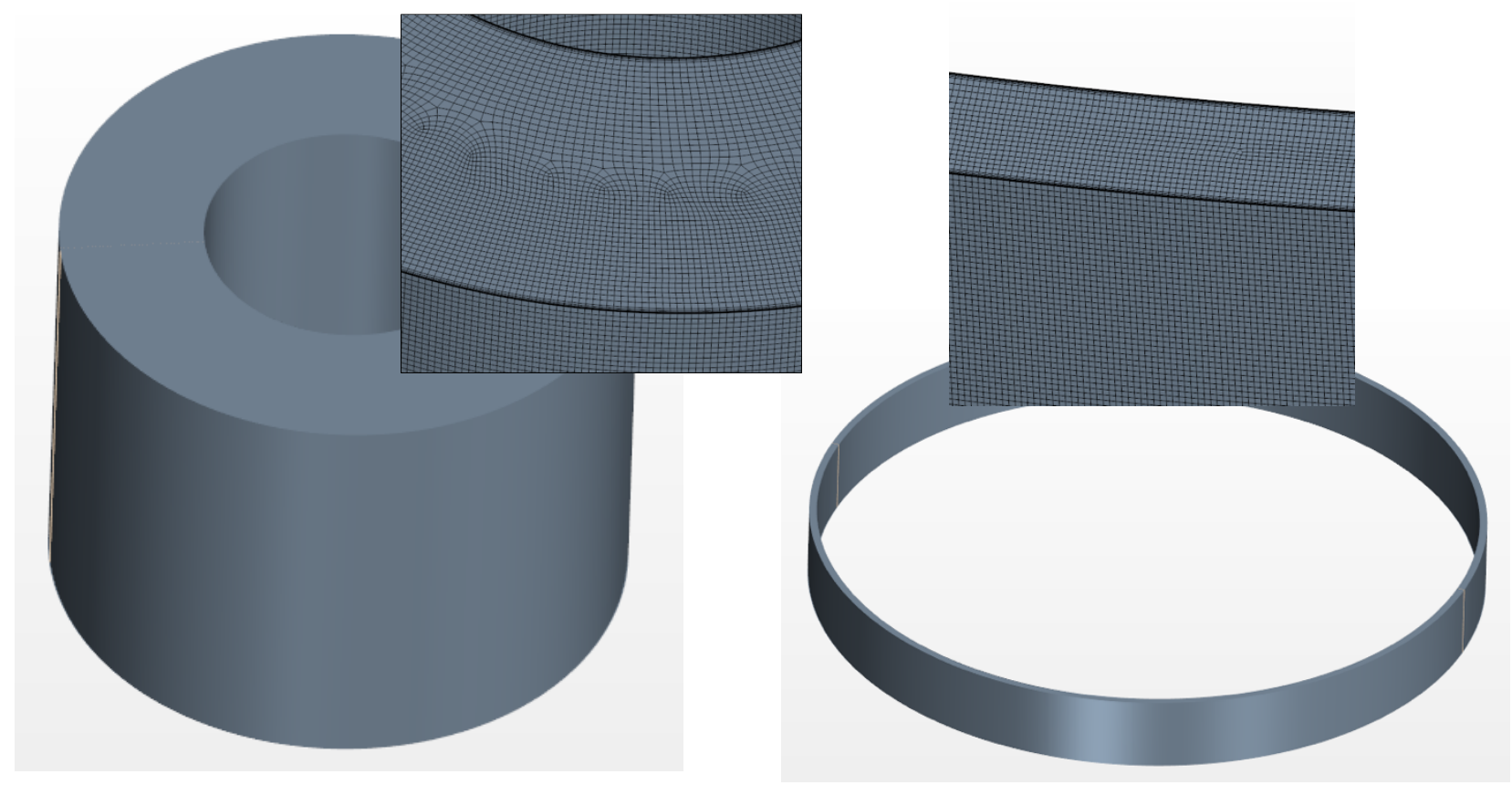

Figure 2. Computational models, geometries, and grids: $R i / d=1$ (left), Ri/d $=44$ (right).

The analytical Couette flow solution, which is used for verification, provides velocity and pressure gradient distributions in the gap. Velocity is shown in Eq. (2), and pressure gradient is shown in Eq. (3) The distributions are functions of the radial coordinate, the inner and outer radii ratio $\eta=R_{i} / R_{0}$, and the rotational speed $\Omega$.

$$
\begin{gathered}
V=-\Omega\left(\frac{\eta^{2}}{\left(1-\eta^{2}\right)} r+\frac{R_{i}^{2}}{\left(1-\eta^{2}\right)} \frac{1}{r}\right) \\
\frac{\partial p}{\partial r}=\rho \frac{V^{2}}{r}
\end{gathered}
$$

\subsection{CONCENTRIC CYLINDERS, COUETTE FLOWS}

The computational models, Models 1 and 2, were run in steady mode for 10,000 time steps to confirm a continuous decline of solution residuals and to verify that no instabilities develop. Figure 3 shows the results from Model $1(\eta=0.5)$ for $R e=55$, which is close to the limit of stable flow. Of the three velocity components, only the radial velocity (V) is non-zero, as shown in the left image of Figure 3. On the right of Figure 3, the velocity and the pressure gradient are compared to the analytical values. A perfect match is noted, confirming the accuracy of the numerical solution in predicting stable shear-dominated flow at low rotational speeds. 


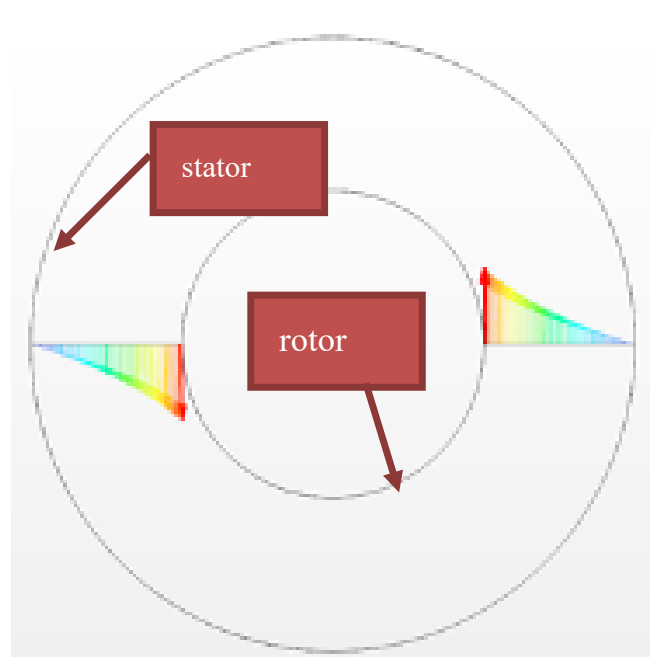

Velocity vectors in gap showing only one radial velocity component

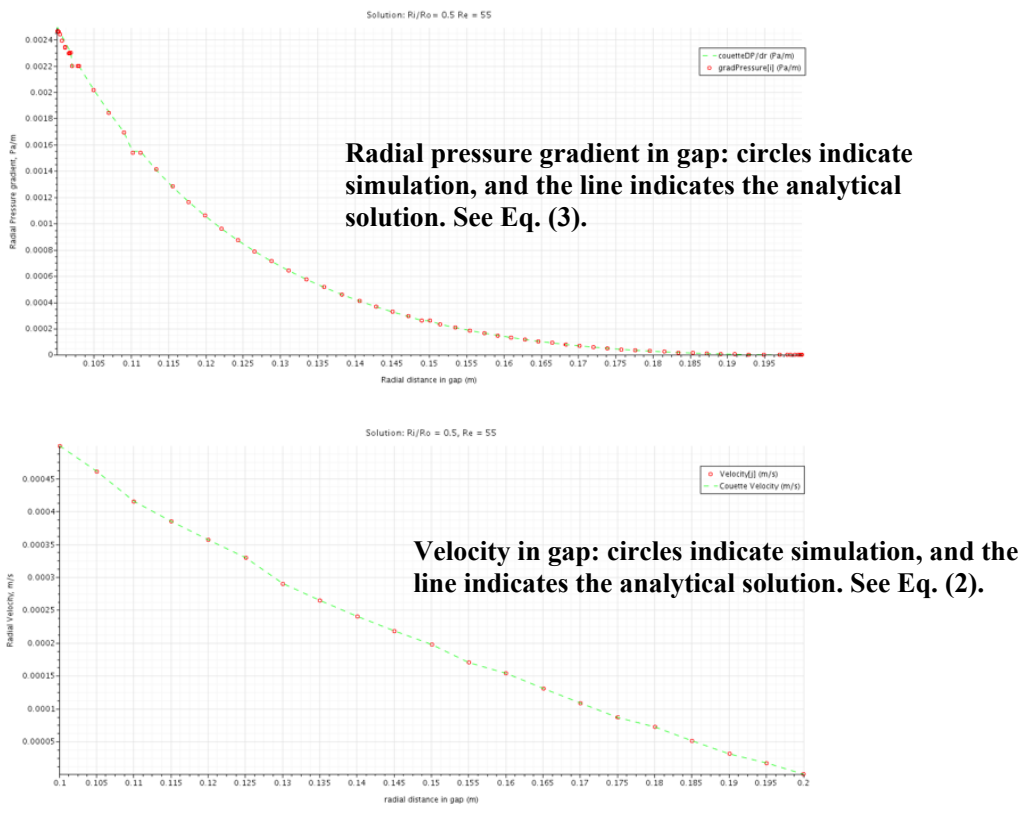

Figure 3. Model 1 results for concentric cylinders $\operatorname{Re}=55, \eta=0.5$.

Figure 4). The narrow gap shear forces stabilize the flow to higher rotational speeds, corresponding to $\operatorname{Re}$ $=250$. As in Model 1, the comparison to the Couette solution is perfect.

With these two calculations, the model verification with analytical solutions was deemed complete. It can be concluded that the balance of forces in the gap (pressure vs. centrifugal) was correcly simulated. At low speeds, the viscous forces dominate and do not allow instabilities to develop.

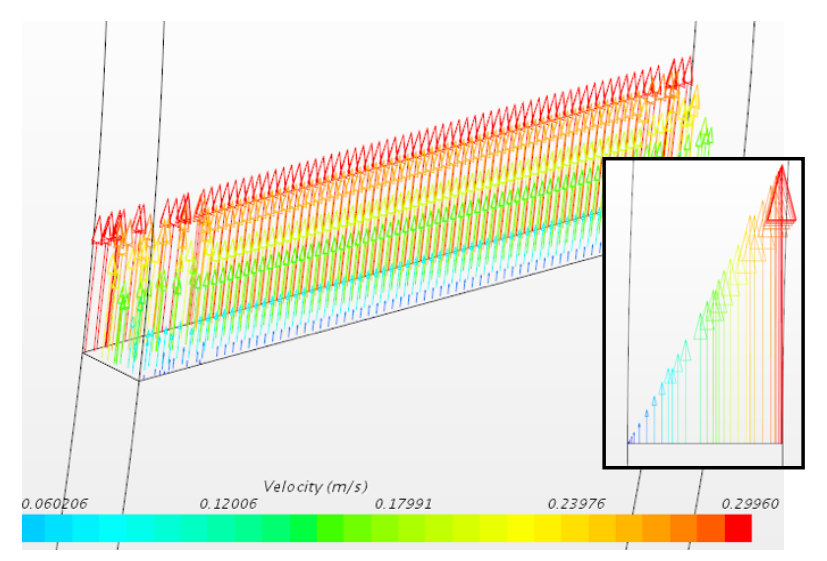

Velocity vectors in gap

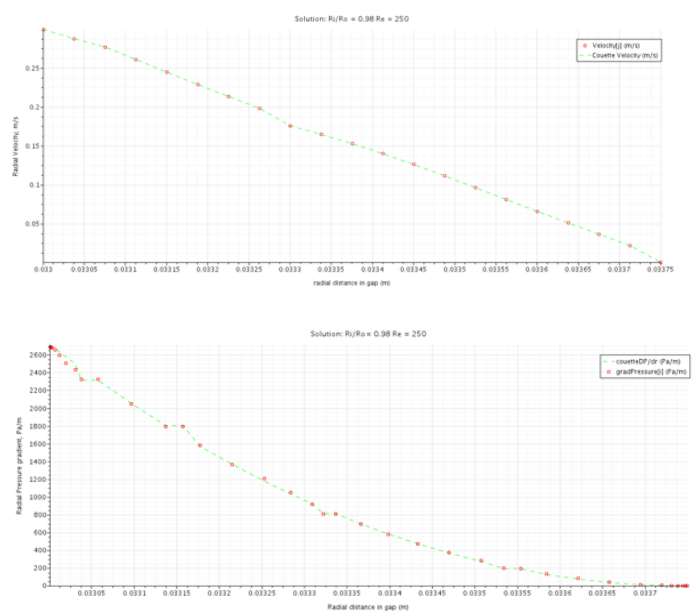

Couette analytical solution vs. numerical data for gap velocity and pressure gradient

Figure 4. Velocity vectors across the gap for Model 2 with $\eta=0.98$ and $\operatorname{Re}=250$.

\subsection{ECCENTRIC CYLINDERS IN STABLE COUETTE FLOWS}

The Couette solution applies to a stable, viscosity-dominated (no instabilities) flow of concentric cylinders. It does not consider the scenario in which one of the cylinders is offset. Such a problem, 
however, is of practical interest for the AMB control. The eccentric case was simulated by assuming an eccentricity $\varepsilon=0.5 / 0.75=0.67$, corresponding to the maximum possible pump shaft shift.
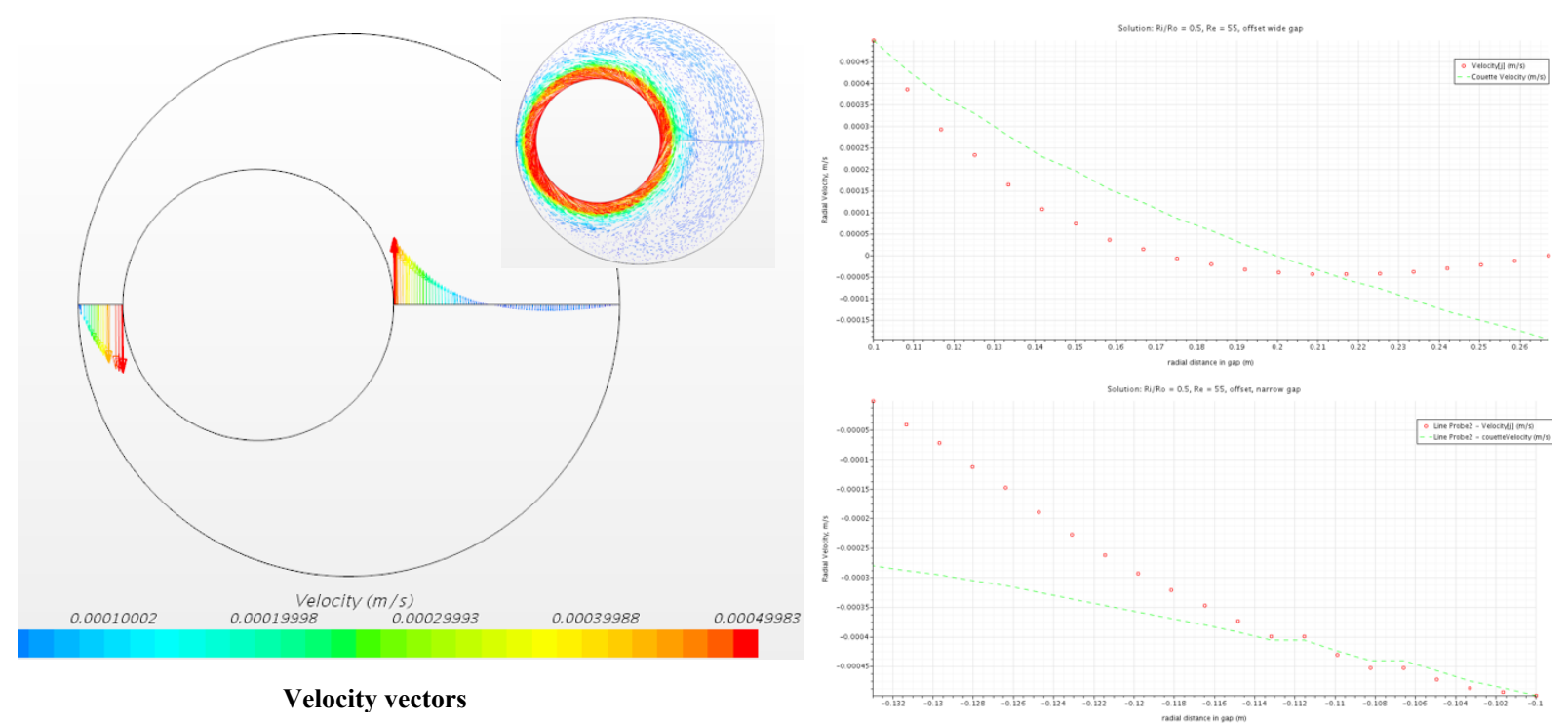

Comparison to Couette solution for the wide (top) and narrow (bottom) sides of the gap

Figure 5. Eccentric case for $h=0.5$ and $\operatorname{Re}=55$.

The offset problem was first computed for Model $1(\eta=0.5)$. The velocities are plotted in

Figure 5. The Re number was kept the same as in the concentric case for consistency. When the rotor shifts, two gaps form - one narrow and one wide. The flow structure in these two gaps is different. In the narrow gap, the flow is very similar to the flow in the symmetric case, having strictly one directional velocity component. However, in the wide gap, a recirculation flow appears that produces a negative radial velocity. The gap velocities are only radial as in the symmetric problem. The Couette solution, which is plotted on the right in

Figure 5, deviates from the numerical result because the boundary condition is not applicable.

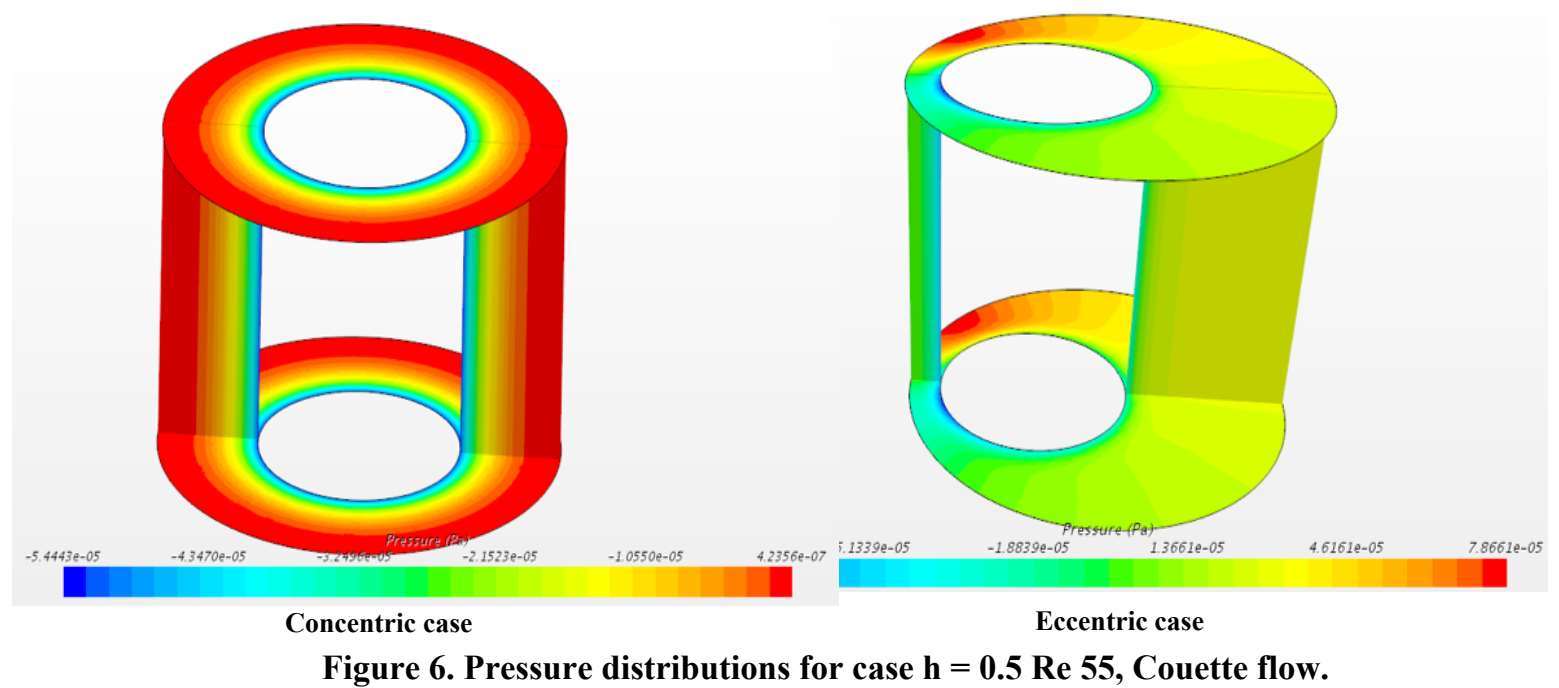


The pressures in the concentric and eccentric problems, however, are quite different, as seen in Figure 6. Hydraulic pressure is the major contributor to the fluid forces acting on the cylinders. In the concentric case, the pressure is radially symmetric, whereas in the eccentric case, high- and low-pressure zones appear on both sides of the inner cylinder.

The narrow gap $(\eta=0.98)$ problem produced identical results (Figure 7$)$. The flow is stable and onedimensional. The recirculation region in the wide gap occupies about half of the gap width, as in the Model 1 (

Figure 5) case. The pressure is asymmetric; it is higher on the side of flow contraction, which results in the formation of a recirculation pattern in the wide gap.
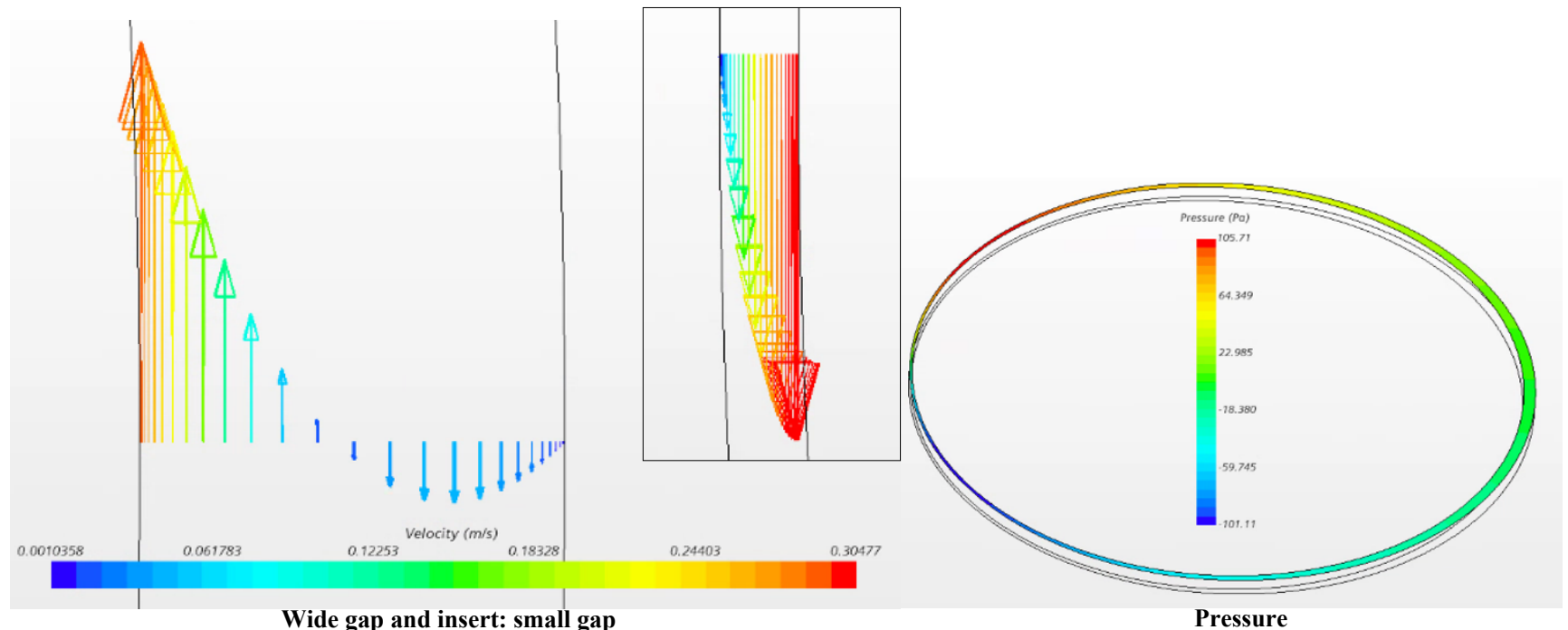

Figure 7. Eccentric case for narrow gap, $h=0.98$ at $R e=250$.

\subsection{TRANSITION TO INSTABILITIES: VORTEX FORMATION}

As the rotational speed of the inner cylinder increases, instabilities appear, causing vortices to develop in the gap. The vortices transport liquid in the radial direction, equalizing the pressure gradient. The critical Re number at which the vortices appear was defined by Recktenwald (A. Recktenwald, 1993) in the function of the inner and outer cylinder ratio, $\eta$. The instabilities begin as detailed in the table below.

Table 2. Instabilities resulting from rotation of inner cylinder

\begin{tabular}{|c|c|c|c|}
\hline \multirow{2}{*}{$\boldsymbol{R}$} & \multirow{2}{*}{ Re (simulation) } \\
\cline { 3 - 4 } & & Concentric & Eccentric \\
\hline 0.5 & 68 & 80 & 150 \\
\hline 0.975 & 261 & 400 & 580 \\
\hline
\end{tabular}

\subsubsection{Concentric Problem $\eta=0.5$ (Model 1)}

The vortices for the concentric problem are shown in

Figure 8. The illustration on the left is from Lueptow (R. Lueptow, 2009). The right plots in Figure 8 show the calculation with Model 1. In the simulation, the gap flow transitions to a vortex structure with a Re number of 80 instead of 68, as predicted by Recktenwald (A. Recktenwald, 1993). In 
the plots on the right-hand side of

Figure 8, velocity is plotted on the left, and vorticity is shown on the right.
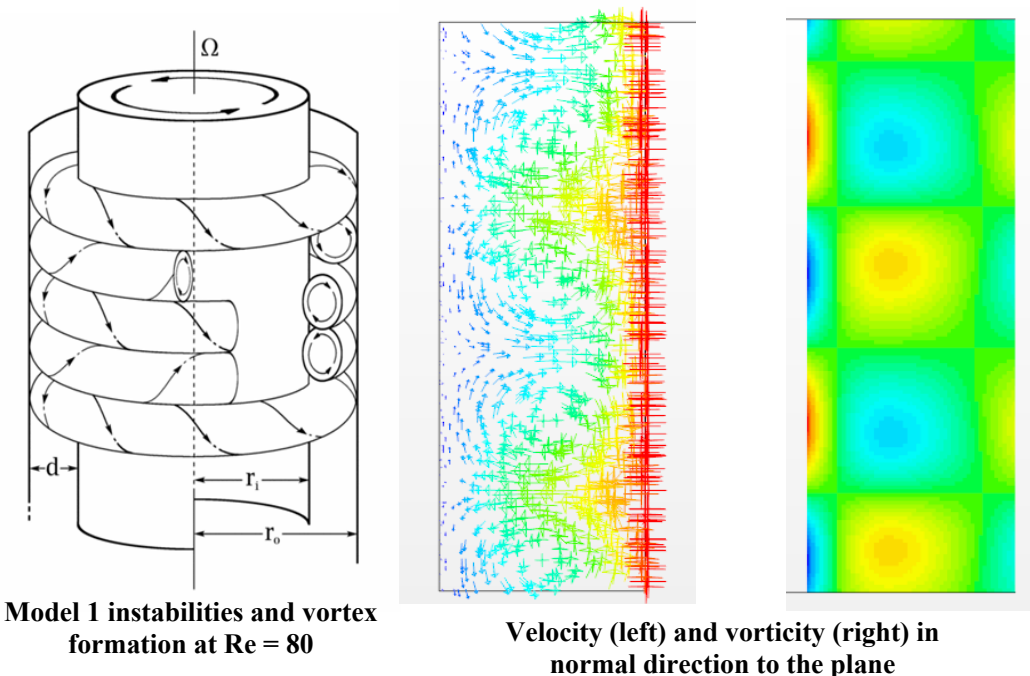

Figure 8. Schematic of counter-rotating axisymmetric vortices of Taylor-Couette flow (R. Lueptow, 2009).

The vorticity is only shown as normal to the plane direction to emphasize the number and direction of vortices more clearly. The vortex structure is more complex than that plotted in

Figure 8 . Within the computed domain, with a length equal to three times the gap ( $3 \mathrm{~d})$, two pairs of vortices form. The wavelength $(\lambda)$ for a single pair is $\lambda=3 \mathrm{~d} / 2=1.5 \mathrm{~d}$, contrary to the results reported by Recktenwald (A. Recktenwald, 1993) $\lambda=2 \mathrm{~d}$ : that is, three individual vortices instead of four. This makes the vortex structure more complex in the simulation, where the vortices are smaller than the gap. An additional flow rotation is noticed next to the inner and outer walls, which is not reported by Recktenwald (A. Recktenwald, 1993). The vortices rotate in opposite directions (against each other), moving liquid in the gap from the inner to the outer cylinder.

\subsubsection{Eccentric Problem $\eta=0.5, \varepsilon=0.67($ Model 1$)$}

The rotational speed of the inner cylinder was gradually increased until a vortex structure in the gap began to appear. The computed critical Re number was 150. Results are shown in Figure 9. The inner cylinder offset stabilizes the flow and delays the instability. The vortices in the narrow gap are barely noticeable. In the wide gap, the vortices occupy only half of the gap, whereas in the other half (closer to the outer cylinder), the flow has low velocity, and vortices do not form.
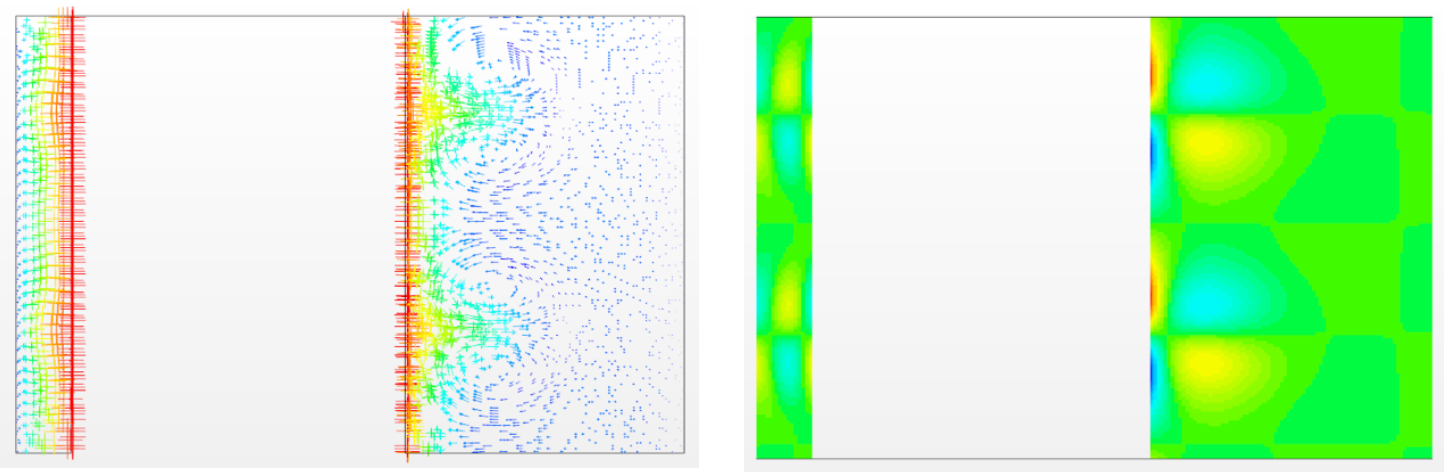

Figure 9. Vortex formation for the eccentric Model 1 problem: velocity (left) and vorticity (right) showing the vortices in the narrow and wide gaps, computed critical $\operatorname{Re}=150$. 
The pressures for the eccentric problem are plotted in Figure 10 for a stable flow on the left $(\operatorname{Re}=55)$, and for an unstable flow at $\mathrm{Re}=150$ on the right. The pressure scale is the same for both plots. It is evident that with increasing rotational speed, regardless of the presence of vortex structure, the pressure load on the inner cylinder increases.
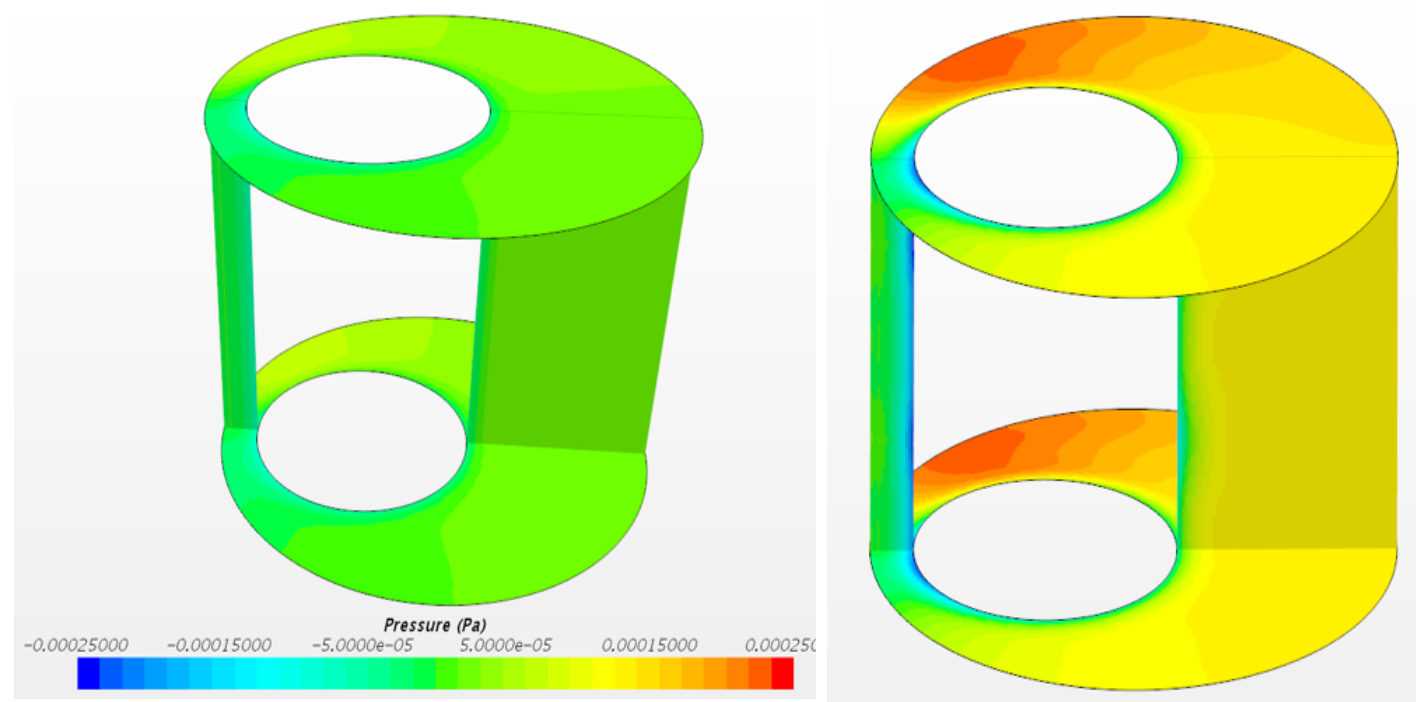

Figure 10. Static pressures for $\operatorname{Re}=55$ stable (left), and $\operatorname{Re}=150$ instability inception (right).

\subsubsection{Concentric Problem $\eta=0.98$ (Model 2)}

According to Recktenwald (A. Recktenwald, 1993), for $\eta=0.98$, the transition to vortical flow should occur at approximately $\mathrm{Re}=261$. The simulations show that at $\mathrm{Re} \approx 400$ and greater, some instability in the gap appears in form of a wavy structure. For lower Re numbers, the flow is stable. Vortices do not explicitly form as they do in the case of $\eta=0.5$ (

Figure 8). Figure 11 shows the result from a transient run after 7 rotations at $R e=400$. The flow structure is wavy (left), as caracterized by radial mounds and valleys. The velocity in the gap follows the Couette profile closely only in the valleys (lower right). The normal to gap vorticity component (upper right) indicates flow rotation. This result resembles a combination of Couette and Taylor vortex flows. Similar to the Model 1 (large gap) result, the wavelength of a single vortex pair is $\lambda=1.5 \mathrm{~d}$ (two pairs in $3 \mathrm{~d}$ domain). The predicted vortices are not fully round, but they occupy the entire gap in the radial direction, which is contrary to the Model 1 , in which additional rotation near the walls is seen. 


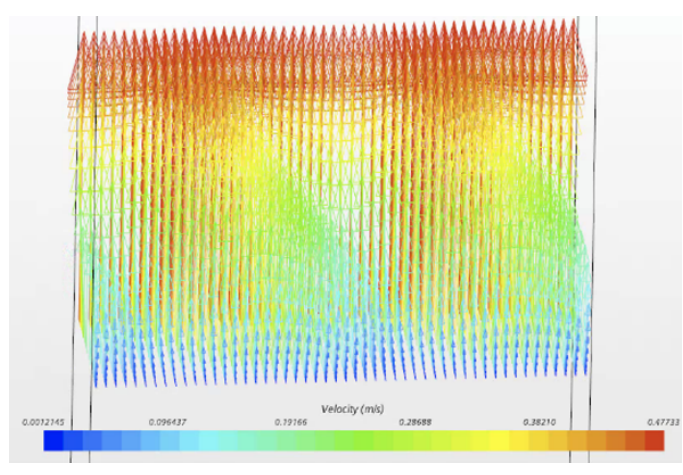

Velocity vectors in gap

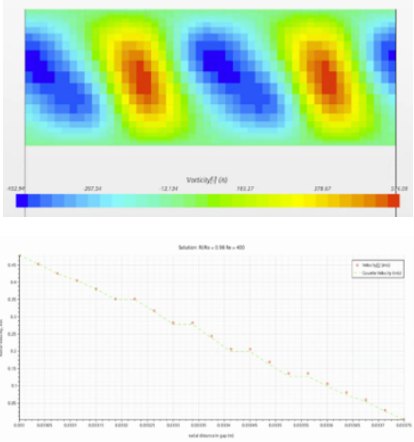

Normal vorticity (top) and Couette velocity comparison (bottom)

Figure 11. Model 2 result at $R e=400$, converged transient simulation with a timestep of 0.001 after $3 \mathrm{~s}$ ( 7 revolutions).

To investigate the problem further, two approaches were analyzed: (1) increasing the rotational speed, and (2) modifying the spatial discretization (computational grid) to improve convergence. A brief summary of this calculation scope is given in Table 3.

Table 3. Summary of meshing sensitivity to modeling the transition to vortices for $\boldsymbol{\eta}=\mathbf{0 . 9 8}$

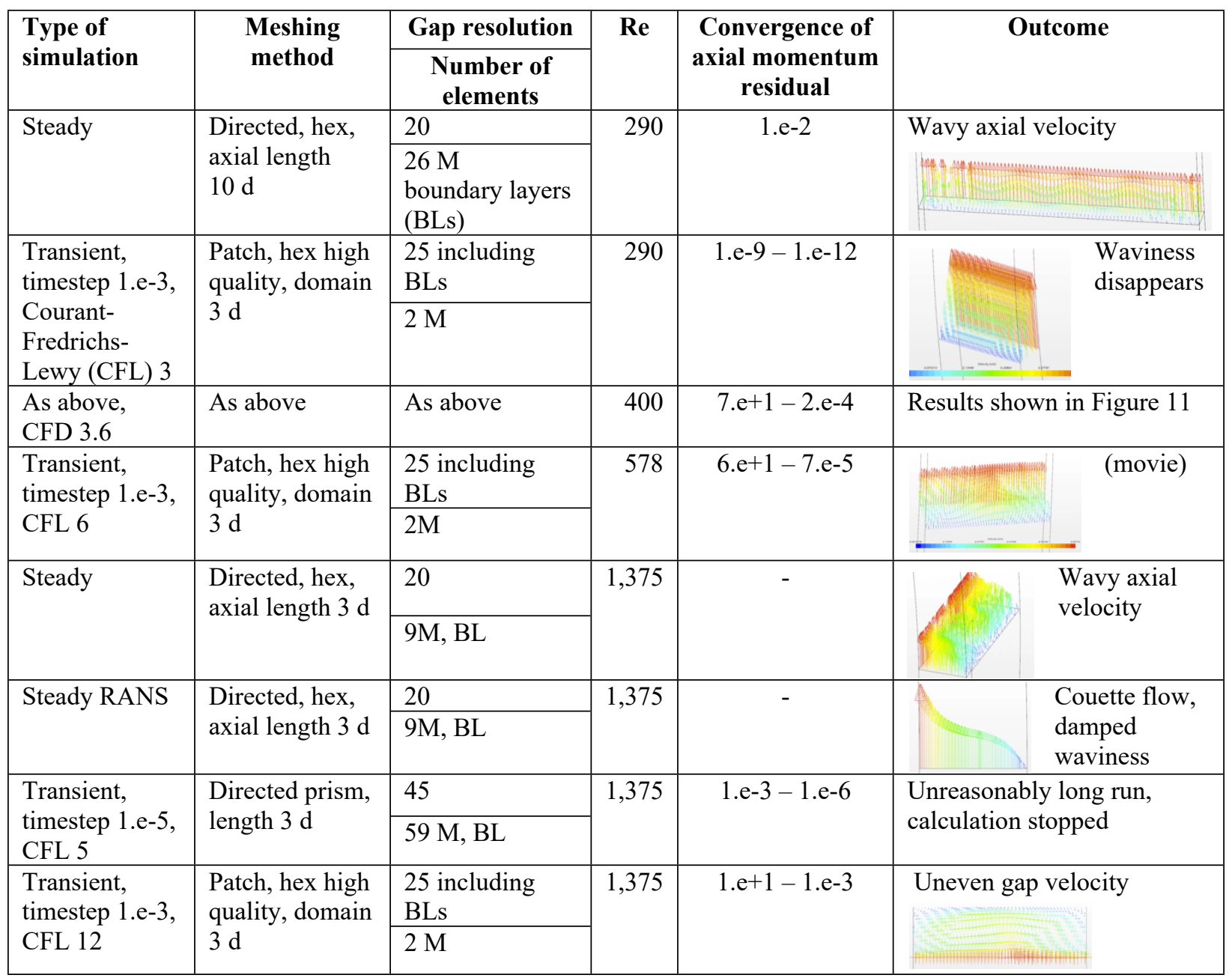


Many meshing techniques were used, resulting in improved convergence. The solution was transitioned from steady to transient, and by varying the number of inner iterations per time step, a good convergence was achieved. The CFL number was controlled and kept below 12, which was considered acceptable for the implicit interative solver that was used. The more accurate (lower residuals) transient solution helped to stabilize the flow at $\operatorname{Re}=290$, as indicated in the first two rows in Table 3), which confirmed that the flow was still in Couette regime.

The Re number was low enough (maximum rotational speed in these simulations was $50 \mathrm{rad} / \mathrm{s}$ ) to assure that the flow remained laminar. The result did not recemble rotating vortices. An axial waviness was noticeable in the gap, which produced mixing, but there was no evidence of stable vortex formation. The waves seem to either travel or fluctuate. This flow behavior continues up to Re numbers way above Recktenwald's $\operatorname{Re}=261$.

To gain a better understanding of the flow structure in small gaps such as those in the salt pump, and to validate the result, the current simulations were compared to those conducted in a similar numerical study (D. Deng, 2007) which included analysis of the flow structure and the Taylor vortex appearance in narrow gaps related to bearing operation. The working liquid was silicone oil, with viscosities an order of magnitude higher than those in the present study. The studied radii ratio $(\eta=0.99)$ is very close to the the radii ratio in this work $(\eta=0.98)$. Calculations were performed using the commercial CFD software $\mathrm{ACE}+$. The results are shown below for three regimes: Couette (Figure 12), Taylor vortex flow (Figure 13), and wavy vortex flow (Figure 14).
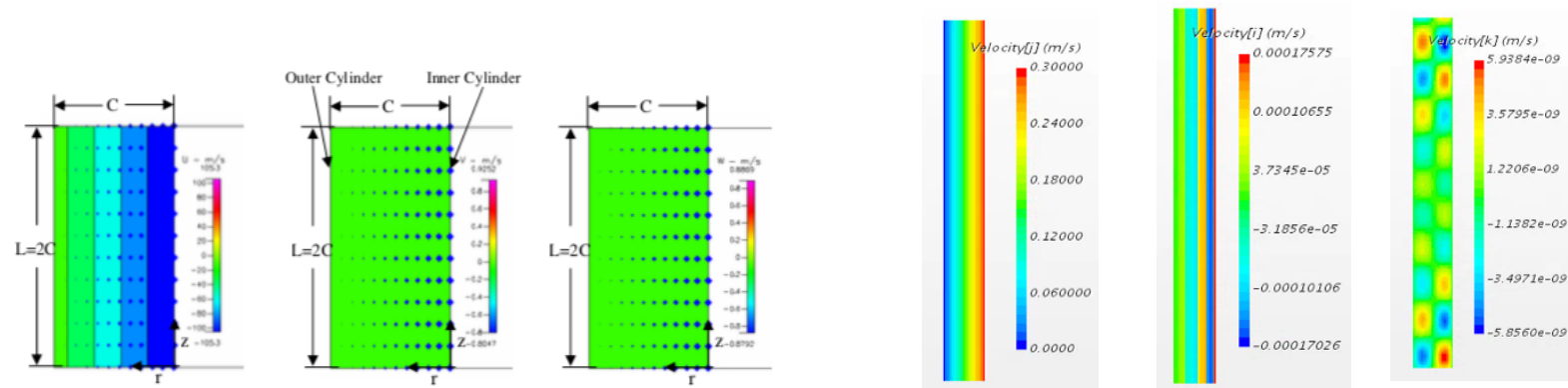

Figure 12. Couette flow regime for narrow gap $\eta=0.99$ (D. Deng, 2007) (left), and $\eta=0.98$ from present study (right). The three velocity components are shown from left to right: normal to the gap, across the gap, and in the axial direction. The result demonstrates stable Couette flow.

The specific parameters for these two computations are given in Table 4.

Table 4. Data for comparative calculations used to validate results for Taylor and wavy flow regimes

\begin{tabular}{|c|c|c|c|c|c|}
\hline \multicolumn{2}{|c|}{ Re number } & \multicolumn{2}{c|}{ Rotation speed, rpm } & \multirow{2}{*}{ Results } & \multirow{2}{*}{ Flow regime } \\
\cline { 1 - 4 } (D. Deng, 2007) & This study & (D. Deng, 2007) & This study & & \\
\hline 283 & 250 & 40,000 & 87 & Figure 12 & Couette \\
\hline 424 & 400 & 60,000 & 138 & Figure 13 & Taylor \\
\hline 708 & $1000-3000$ & 100,000 & $477-1092$ & Figure 14 & Wavy \\
\hline
\end{tabular}

At low rotational speed, the flow is dominated by viscous forces and is very regular, with only radial (normal to a plane across the gap) velocity component. In Figure 12, note the scale on axial velocities and across-gap velocities. The computations were carried out for slightly different Re numbers, but they were within the Couette regime. The domain used in the present calculation is $10 \mathrm{~d}$, whereas in Deng (D. Deng, 2007), the domain is $2 \mathrm{~d}$. Results are practically identical, thus confirming the Couette flow structure in the analyzed Re range. 

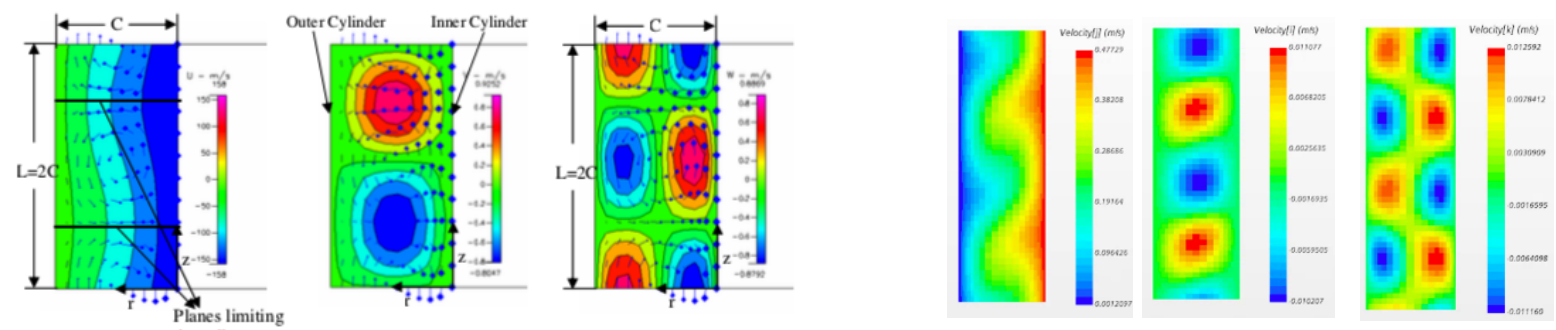

Figure 13. Taylor vortex flow regime for narrow gap $\eta=0.99$ (D. Deng, 2007) (left), and $\eta=0.98$ from present study (right). The three velocity components are shown from left to right: normal to the gap, across the gap, and in the axial direction.

In this work, the instabilites appear at approximately $\mathrm{Re}=400$, and they continue to be seen at much higher Re numbers. The code-to-code comparison was initiated to confirm this result. Deng (D. Deng, 2007) concludes that at $R e=410$, the flow structure transitions to Taylor vortices. Figure 13 shows comparison of the flow structure at Re numbers deemed by Deng to represent Taylor vortex flow, and at $\mathrm{Re}=400$ from this study. The simulations show very close results. Deng's axial domain was $2 \mathrm{~d}$, whereas in this simulation, it was $3 \mathrm{~d}$. Deng predicts one pair of vortices: the vortex wave length is $\lambda=2 \mathrm{~d}$; however, in this simulation, two pairs are visible, resulting in $\lambda=3 \mathrm{~d} / 2=1.5 \mathrm{~d}$, which is likely the major discrepancy. The normal to the gap velocity component shows waviness, but the vortices are not that well expressed as those shown in

Figure $8(\eta=0.5)$. The results from these two calculations deviate from the results of Recktenwald's analysis (A. Recktenwald, 1993) by a factor of approximately 1.7, which might be a result of the purely analytical derivation used by Recktenwald, which is contrary to the numerical approach used in this study and in Deng's work (D. Deng, 2007).

With a further increase in the Re number, the flow transitions to a wavy votex flow. Deng concludes that the transition occurs at $\operatorname{Re}=438$. Note that the Taylor flow occurs in a very narrow range, between $\operatorname{Re}=$ 410 and $=438$. In Figure 14, a comparison is made using a much higher Re number (see Table 4), and it seems that the flow maintains the same structure as that shown in Figure 13. There is no qualitative difference in the velocity patterns, which to some extent confirms the observations made above: that the flow in a narrow gap transitions to a wavy vortex flow directly from the regular Couette flow. It is interesting to note that at high speeds, the wavelength of vortices in the present simulation increases to $\lambda$ $=3 \mathrm{~d}$, and only a single vortex pair is seen in the gap, whereas in Deng's analysis, the wavelength is invariant in relation to the speed.

Based on this limited comparison to results from other studies, it can be concluded that the developed model produces repeatable results and can be used for analysis of flows in the gap between the rotor and stator of a molten salt pump.
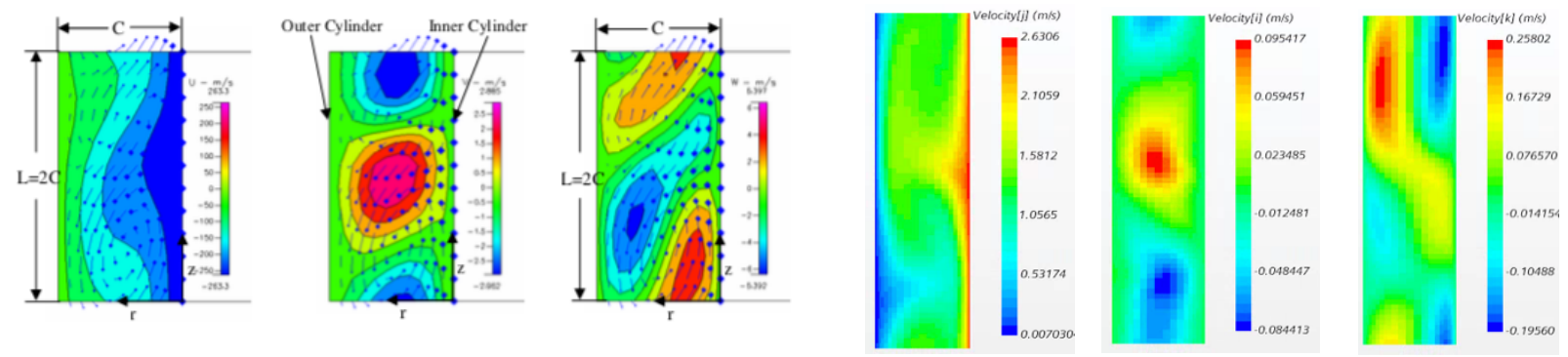

Figure 14. Wavy vortex flow regime for narrow gap $\eta=0.99$ (Deng, 2007) (left), and $\eta=0.98$ from present study (right). The three velocity components are shown from left to right: normal to the gap, across the gap, and in the axial direction. 


\subsubsection{Eccentric Problem $\eta=0.98$ (Model 2)}

For the eccentric case, the transitional Re number was sought by gradually increasing the inner cylinder rotational speed, starting with the stable problem shown in Figure 7. Some waviness starts to appear at approximately $\operatorname{Re} 550$ and becomes more expressed at $\operatorname{Re}>580$. Results for $\operatorname{Re}=550$ are shown in Figure 15. The velocity component across the gap is most indicative for the appearance of vortices. As seen in the right-hand portion of Figure 15, no clear vortices are visible at $\mathrm{Re}=550$. The magnitude of the velocity component is very low, which means that the flow structure is still dominantly one-dimensional, or only radial velocity, as in the Couette regime.
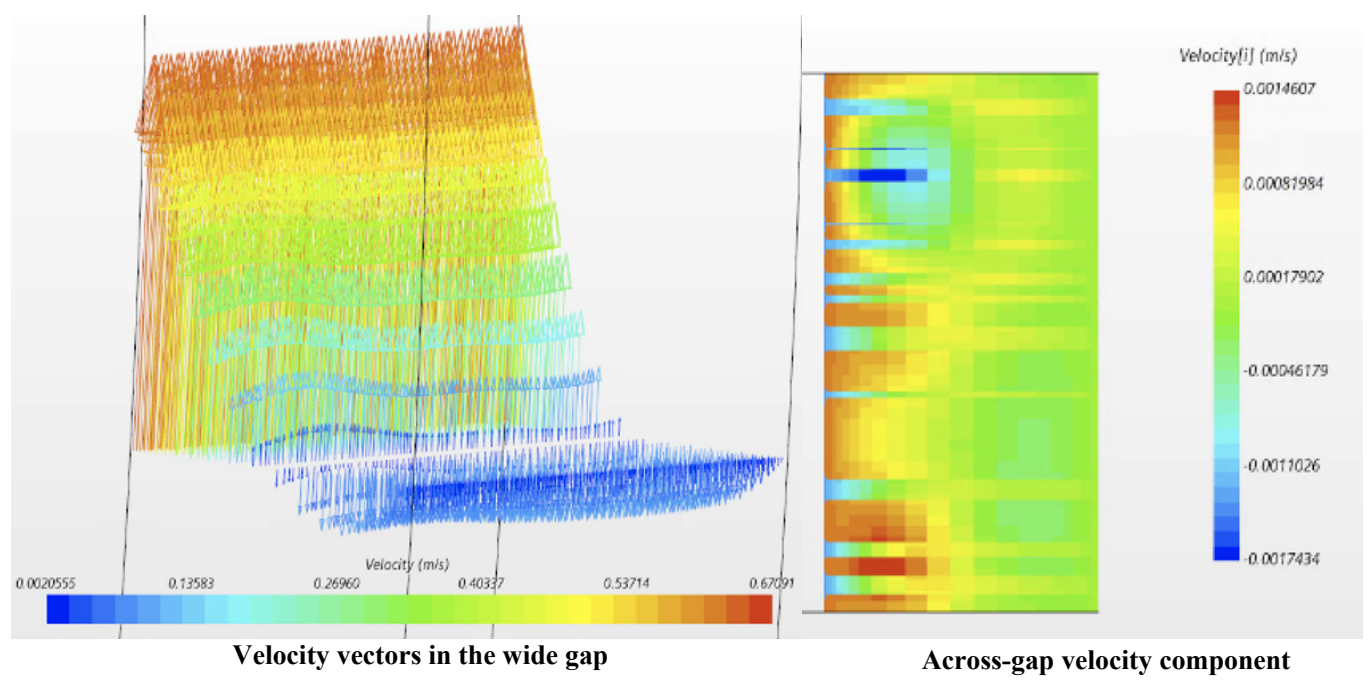

Figure 15. Eccentric case, narrow gap $(\eta=0.98)$ beginning of transition to vortex wavy flow at $\operatorname{Re}=550$.

At about $210 \mathrm{rpm}(\operatorname{Re}=580)$, the waves augment, and vortices form in the gap, but only this only occurs on the wide side. In the narrow gap, the flow is still in the Couette regime, as shown in

Figure 16. The magnitude of these vortices is low, and they do not travel in the axial direction. Based on an analogy with the concentric case, this regime should correspond to Taylor flow. Two vortex pairs form, as in the concentric case, resulting in the same wavelength. It appears that the wavelength does not depend on the offset of the inner cylinder and is only dependent on the rotational speed. This observation still must be confirmed with analyses of offset problems at higher angular velocities. 


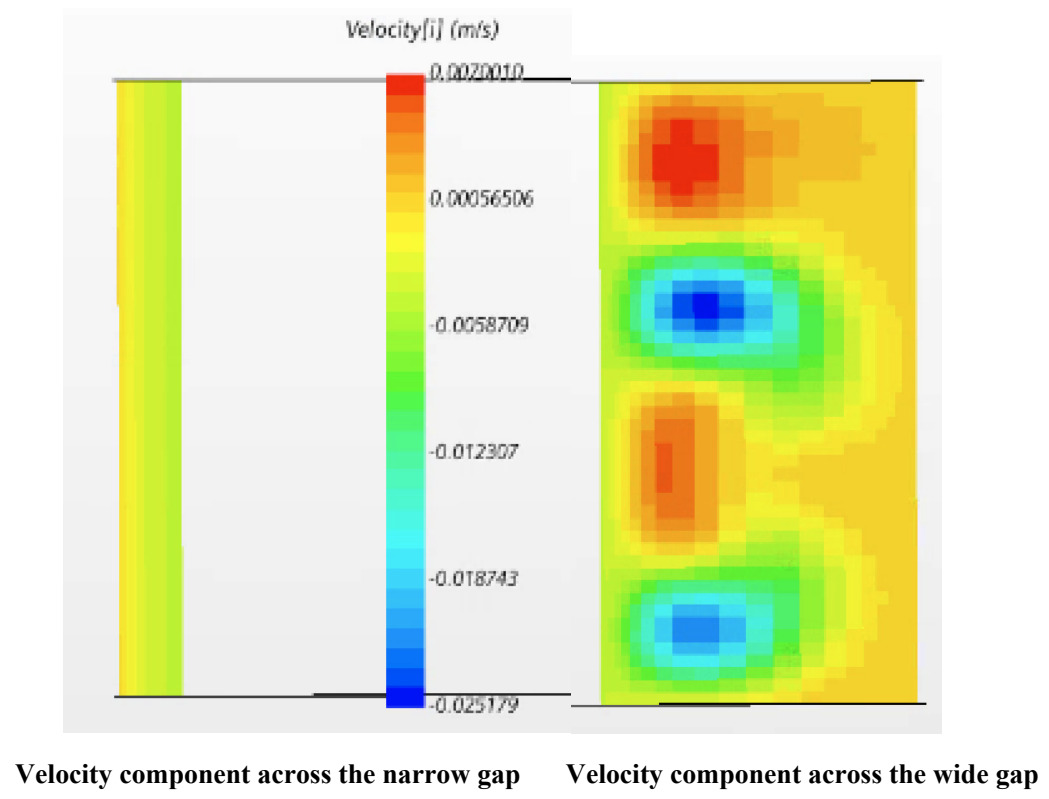

Figure 16. Inception of Taylor vortices for $\eta=\mathbf{0 . 9 8}$ and $\mathbf{R e}=\mathbf{5 8 0}$.

Another specific feature is that the vortices do not occupy the entire gap, but rather, they are attached to the moving cylinder. To aid in visualization of the flow structure, a plot of the velocity vectors is provided in Figure 17. The maximum velocity next to the moving cylinder wall is trimmed to a lower value to allow for a clearer picture of the gap velocity distribution. The wavy structure of flow is propagating across the entire gap, but it changes direction the next to the wall region, where vortices exist, whereas it remains negative (reversed direction) in the second half of the gap next to the stationary wall. The magnitude of the reversed flow varies, creating the appearance of waves in the entire gap. Although the appearance is different, the eccentric problem flow structure has no qualitative differences when compared to the concentric case. The same waves begin to appear at slightly higher speeds, but otherwise, the flow pattern remains the same.

Based on these simulations, the analysis of the flow at relatively low rotational speeds up to approximately $200 \mathrm{rpm}$ is considered complete. The flow in gaps, which is similar to that in the molten salt pump, transitions to a wavy flow with a complex vortex structure. The eccentricity does not affect the structure; it only stabilizes the flow to higher speeds. Analyses will continue with simulations of flows at speeds closer to what actually occurs 300-1800 rpm and they will focus on narrow gaps with variable eccentricity. 


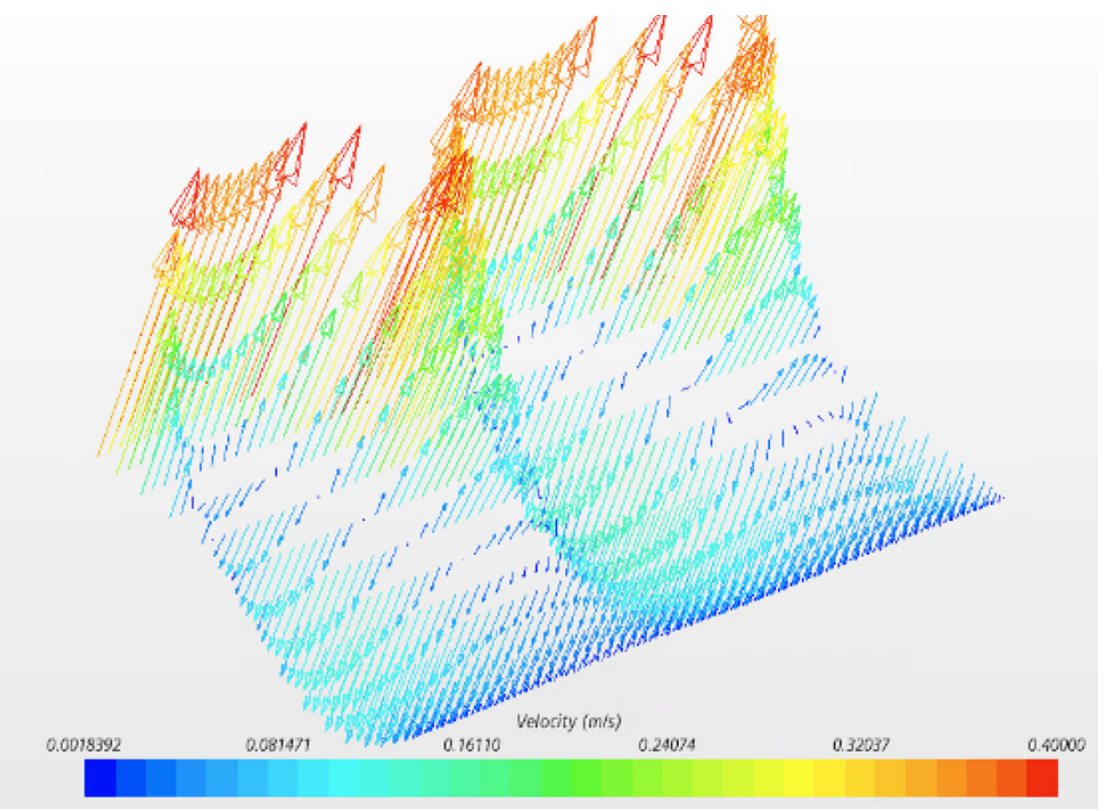

Figure 17. Flow structure in wide gap, $\operatorname{Re}=580 \eta=0.98$. Maximum velocity is clipped to a lower value of 0.4 to better illustrate the flow direction and the wavy structure. 


\section{FLUID FORCES ACTING ON THE INNER CYLINDER}

The goal of these simulations is to compute the fluid forces acting on the inner cylinder to assist in the development of an active magnetic bearing control algorithm. The inner cylinder represents a surface immersed in the liquid rotating at a constant speed. The surface is subjected to two types of forces: a hydrodynamic pressure force, and a wall shear force. Typically, the hydrodynamic pressure is dominant, because it has a higher magnitude.

The AMB control mathematical representation uses a rigid body dynamics mechanical model in the center of mass coordinates. The fluid forces contributing to offset the inner cylinder (pump rotor) from its centered position are acting in a plane perpendicular to the cylinder axis. It was assumed that this is the $\mathrm{X}-\mathrm{Y}$ plane, and the fluid forces were calculated for this plane.

In the CFD model, each surface was discretized with facets $(f)$, which are small planes forming the computational element. The force $(F)$ on a continuous surface was computed as a sum of fluid forces acting on each individual facet:

$$
F=\sum_{f}\left(F_{f}^{\text {pressure }}+F_{f}^{\text {shear }}\right) \cdot \boldsymbol{n},
$$

where

$$
\begin{aligned}
& F_{f}^{\text {pressure }} \text { and } F_{f}^{\text {shear }} \text { are the force vectors from pressure and shear on a facet, and } \\
& \boldsymbol{n} \text {-is a user-specified direction for which the total force is projected. }
\end{aligned}
$$

In this instance, the force was projected on the $X$ and $Y$ directions of the plane orthogonal to the axis of rotation.

The pressure force component was computed as follows:

$$
F_{f}^{\text {pressure }}=\left(p_{f}-p_{\text {ref }}\right) a_{f}
$$

where $p_{f}$ is the hydrodynamic pressure, $p_{r e f}$ is a reference pressure, and $\boldsymbol{a}_{f}$ is the facet area vector.

The shear force was computed as

$$
F_{f}^{\text {shear }}=-T_{f} \cdot a_{f},
$$

where $\boldsymbol{T}_{f}$ is the shear stress tensor, defined as

$$
T=\mu\left(\nabla v+(\nabla v)^{T}\right)-\frac{2}{3} \mu(\nabla \cdot v) I,
$$

where:

$$
\begin{aligned}
& \mu \text { is the dynamic viscosity of liquid, and } \\
& v \text { is velocity. }
\end{aligned}
$$

For incompressible fluids, as in this simulation, the second term in Eq. (7) is zero.

The fluid forces are dynamic and were computed in function of time. They were also computed by using an axial periodic condition, so they must be rescaled to the real inner cylinder length in order to produce the total forces acting on the cylinder. 


\section{COMPUTING OF FORCES IN HIGH-SPEED ROTATIONAL FLOWS: CONCENTRIC CYLINDERS}

This section presents the evaluations of flows in the gap at high rotational speeds. The molten salt pump is designed for speeds of up to 2,400 rpm $(40 \mathrm{~Hz})$. The simulations were carried out with $5 \mathrm{~Hz}(300 \mathrm{rpm})$ increments. The maximum speed was assumed to be reached for 8 seconds, resulting in a linear ramp up speed of $300 \mathrm{rpm} / \mathrm{sec}$.

\subsection{INNER CYLINDER ROTATIONAL SPEED OF 300 RPM (RE = 840)}

The problem was simulated by increasing the speed from 0 to $300 \mathrm{rpm}(5 \mathrm{~Hz})$ for 1.0 second. Then the calculation was carried out for another 4 seconds at a constant speed to allow the flow to stabilize and reach a steady behavior. This corresponds to 25 rotations. To hold the CFL below 10, timesteps of 0.0005 seconds were used, and 10,000 timesteps were computed for $5 \mathrm{~s}$ of real time. Each timestep was converged for 40 inner loops of the iterative solver to lower the momentum residuals by at least three orders of magnitude. The simulation took several days to complete on 16 compute processes.

This speed range is characterized by flow transition from viscous to wavy vortex flow, and the structure changed rapidly from Couette to Taylor and then to wavy. The force is shown in Figure 18 from a run that uses a domain size of $6 \mathrm{~d}$ ( 2 wavelengths). This domain size was found to be more representative for the actual force, which is further analyzed in Section 4.2 below.

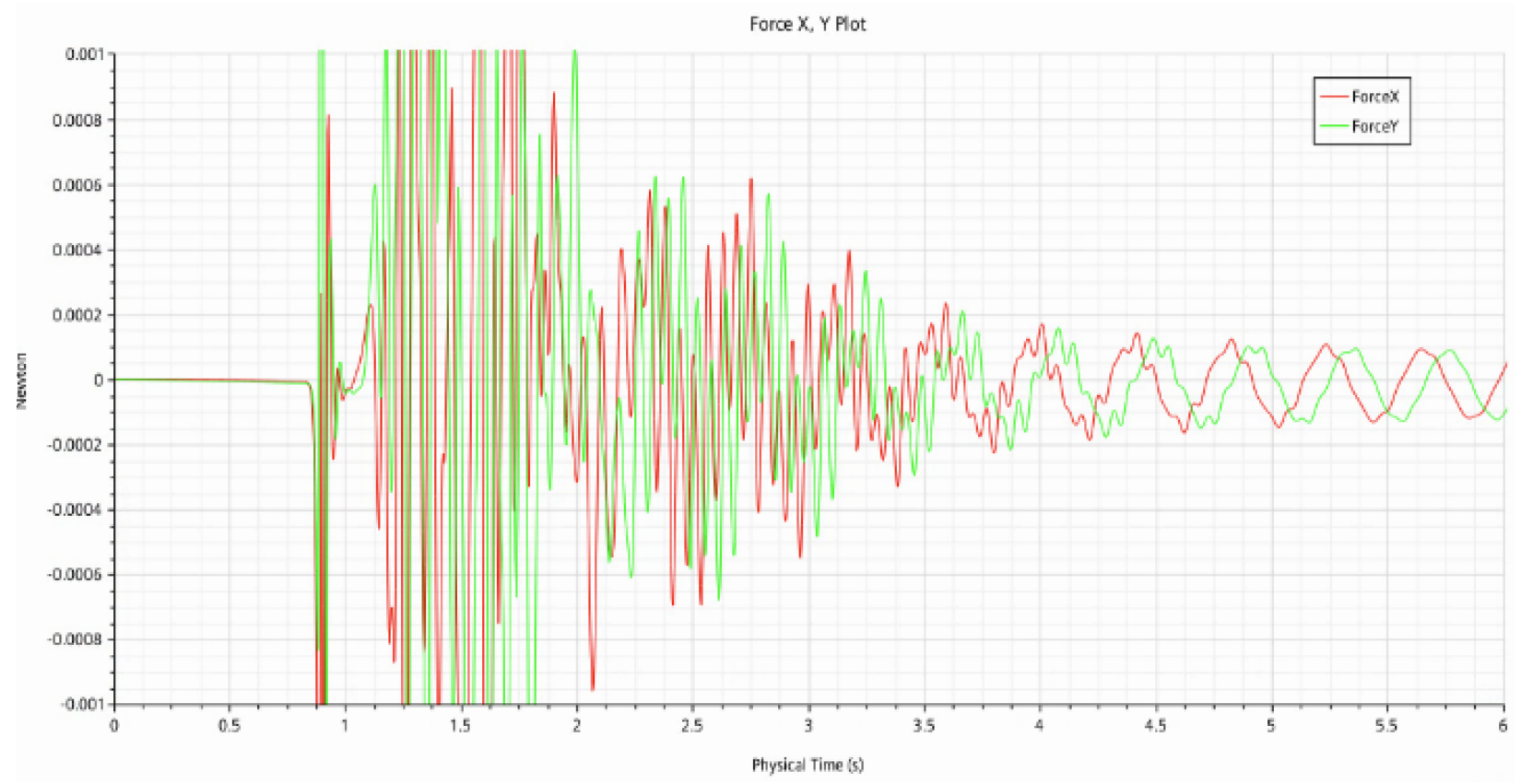

Figure 18. Forces on the inner cylinder for a speed of $300 \mathrm{rpm}$ and an axial domain of $6 \mathrm{~d}$.

As explained above, the velocity linearly increases from zero to $300 \mathrm{rpm}$ in one second. The force is practically zero in the Couette regime. At around $260 \mathrm{rpm}(0.85 \mathrm{~s})$, the force begins to develop. This velocity corresponds to a wavy flow structure. Taylor vortices were previously calculated to appear at 138 $\mathrm{rpm}$. The corresponding speed is attained at around $0.4 \mathrm{~s}$, which means that during the Taylor vortex flow, the forces are also not noticeable. For the first second of transient at a constant speed of 300 rpm (1-2 s) the force does not follow a particular pattern. In the remainder of the calculated period, the force takes on an oscillatory behavior, and toward the end of the period $(6 \mathrm{~s})$ it stabilizes to a clear wavy profile. The initial unstructured force period of $1-2$ s could be attributed to a flow transition, from stable to wavy 
vortex flow. The used ramp-up speed of $300 \mathrm{rpm} / \mathrm{s}$ has a shorter timescale than the periods of flow restructuring and force development.

The force on the inner cylinder, decomposed on $X$ and $Y$ coordinates (Figure 18), changes direction with a frequency of about $2.5 \mathrm{~Hz}$ over a period of $0.4 \mathrm{~s}$. This frequency corresponds to half of the cylinder rotational speed $(5 \mathrm{~Hz})$, which is equivalent to the average gap flow velocity. This indicates that the force is caused by rotating vortices carried by the flow at an average speed of $2.5 \mathrm{~Hz}(150 \mathrm{rpm})$. There is a tendency of force decay, but the calculated period is short and does not allow the decay rate to be determined.

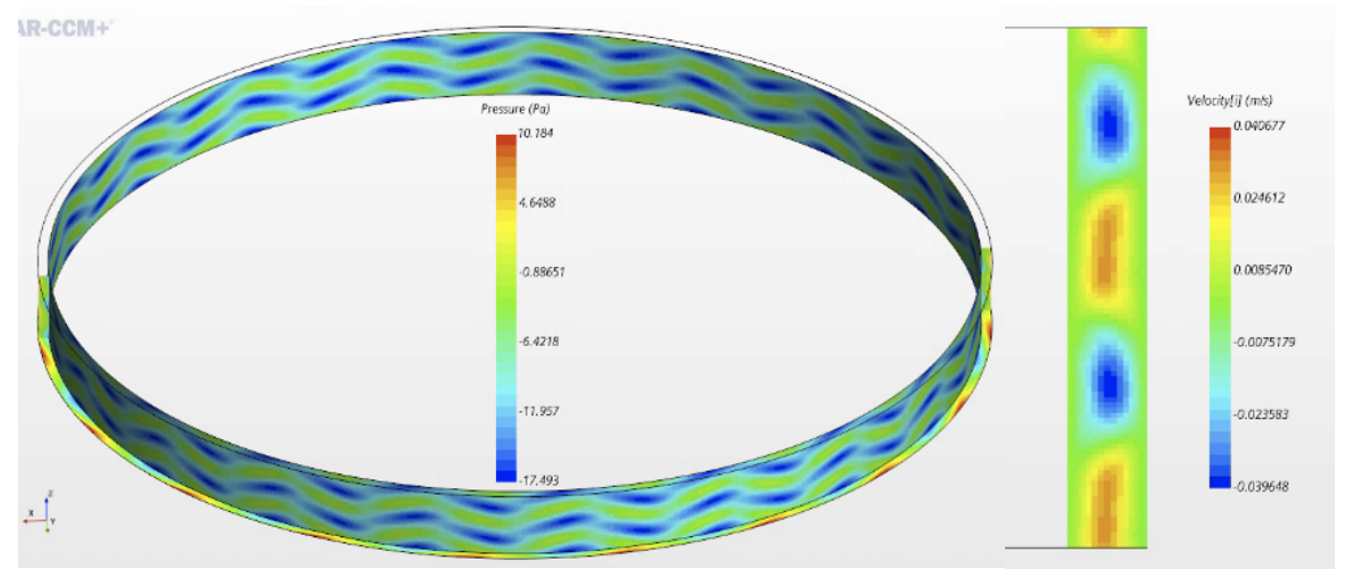

Figure 19. Pressure on the inner wall (left) and the radial velocity in the gap (right) for a speed of $300 \mathrm{rpm}$, domain $6 \mathrm{~d}$.

To illustrate the flow structure at $300 \mathrm{rpm}$, the pressure on the rotating cylinder (left) and the cross gap velocity (right) are plotted in Figure 19. Two pairs of vortices are noticeable, as indicated by their pressure imprints on the wall. The vortices do not seem to travel in the axial direction; rather, they stay confined within the computed domain of two wave lengths $(2 \lambda)$.

\subsection{ROTATIONAL SPEEDS OF 600 RPM, OR $10 \mathrm{HZ}(\mathrm{RE}=1,680)$ : EFFECT OF THE AXIAL DOMAIN SIZE}

A speed of $600 \mathrm{rpm}$ was achieved by increasing the speed of the rotating cylinder from 300 to $600 \mathrm{rpm}$ for one second. This speed range is entirely in the wavy regime and is more suitable for use in analyzing the flow structure and the force dependence on the axial domain size. Three domains were simulated with 3,6 , and $9 \mathrm{~d}$ (gap sizes) axial lengths. They correspond to 1, 2, and $3 \lambda$. The same mesh resolution was used for the grids, thus minimizing the discretization effect. 


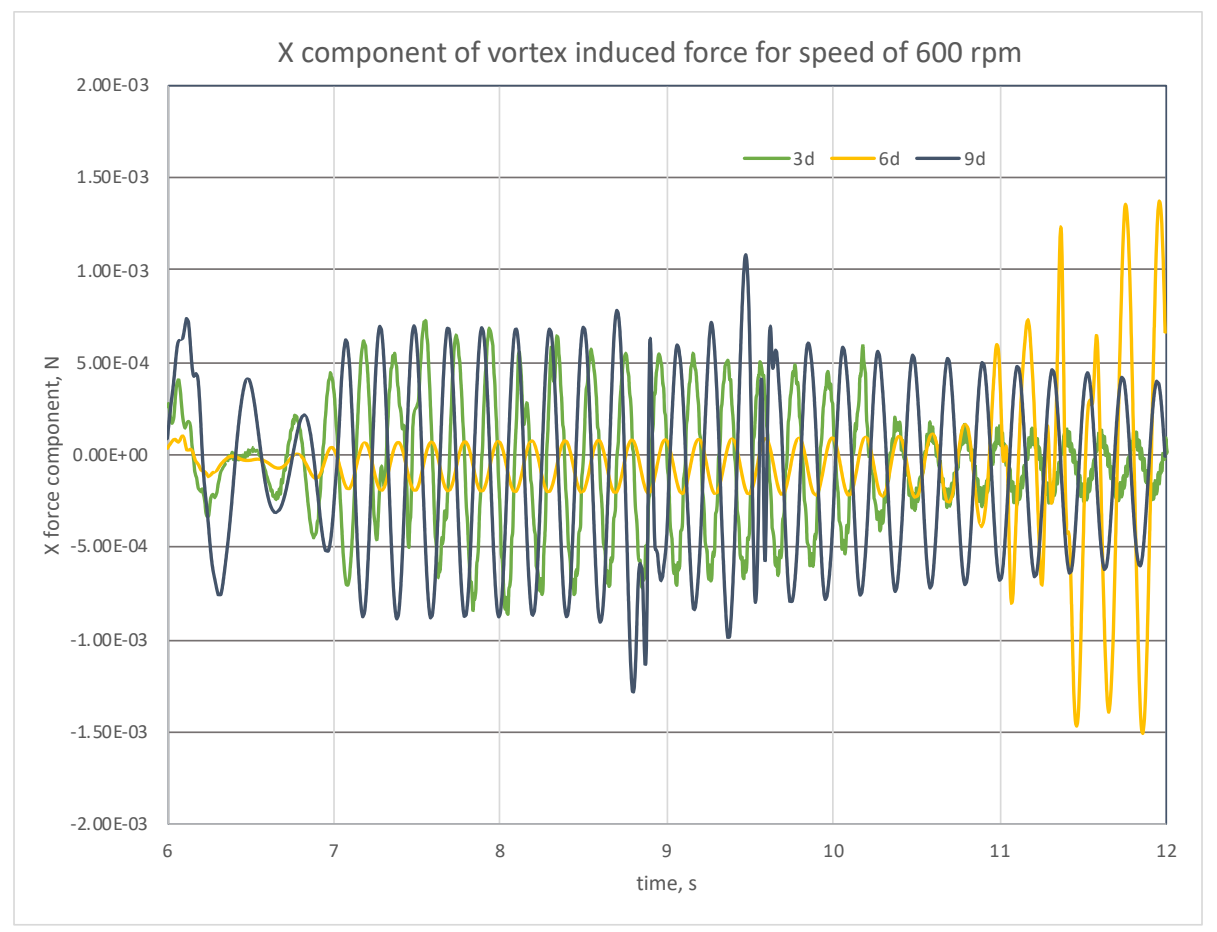

Figure 20. $X$ component of vortex-induced force for three axial domain sizes $-3,6$, and $9 \mathrm{~d}-$ plotted on the same scale for comparison. During the first second (6-7 s), the speed increased from 300 to $600 \mathrm{rpm}$, and then it remained constant for 5 seconds.

Figure 20 combines the results from the three problems by showing the force projection on the $X$ axis, which is orthogonal to the rotating cylinder. In all three cases, the forces oscillated with the same frequency of $5 \mathrm{~Hz}$, corresponding to the vortex rotational speed. However, the magnitude was different, and some phase shift was noticed. The $6 \mathrm{~d}$ case initially had the lowest magnitude, which grew toward end of the period, whereas the 3 and $9 \mathrm{~d}$ cases had similar magnitudes in the initial period. The magnitude varied over time, but no dependence on the domain size was evident. Also, some time shift was seen that was likely caused by restructuring during the initial period of flow, after the velocity remained constant $(7 \mathrm{~s})$. This result indicates that both the force magnitude fluctuation and the time shift have a numerical origin. Because the force magnitude is insignificantly low for the practical problem of magnetic bearing control, no further investigation of the numerical instability was carried out. Based on this result, it was concluded that the actual domain, which is about $220 \mathrm{~d}$, will not generate vortex-induced forces of merit for the magnet control.

An illustration of the flow structure for the cases described above is provided in Figure 21. The pressure on the inner wall is shown on the left. Two features are noticeable. The $9 \mathrm{~d}$ domain does not contain three vortex pairs as expected; instead, it has two vortex pairs, as in the $6 \mathrm{~d}$ case. The vortices simply widen and take the entire space. The axial wavelength for the 3 and $6 \mathrm{~d}$ domains is $3 \lambda(3 / 1=6 / 2=3)$, whereas for the $9 \mathrm{~d}$ problem, the axial wavelength is $9 / 2=4.5 \lambda$. Another finding is that the waves stretch more in the azimuthal direction relative to the $300 \mathrm{rpm}$ case (Figure 19). For the $300 \mathrm{rpm}$ case, 18 waves are distinguishable, whereas only 12 waves can be counted for the $600 \mathrm{rpm}$ case. As expected, the axial domain size does not affect the azimuthal vortex wavelength. This peculiarity was monitored further at higher speeds.

The right portion of Figure 21 shows a single particle track for the three cases. While the $3 \mathrm{~d}$ domain seems narrow, and the particle often leaves the domain, the 6 and $9 \mathrm{~d}$ cases fully encompass the particle track. This confirms the understanding that there is no flow motion in the axial direction, except for some 
light deviation from a perfect trajectory (as seen in the Couette regime) within the $6 \mathrm{~d}$ axial space. The vortices do not travel in the axial direction.

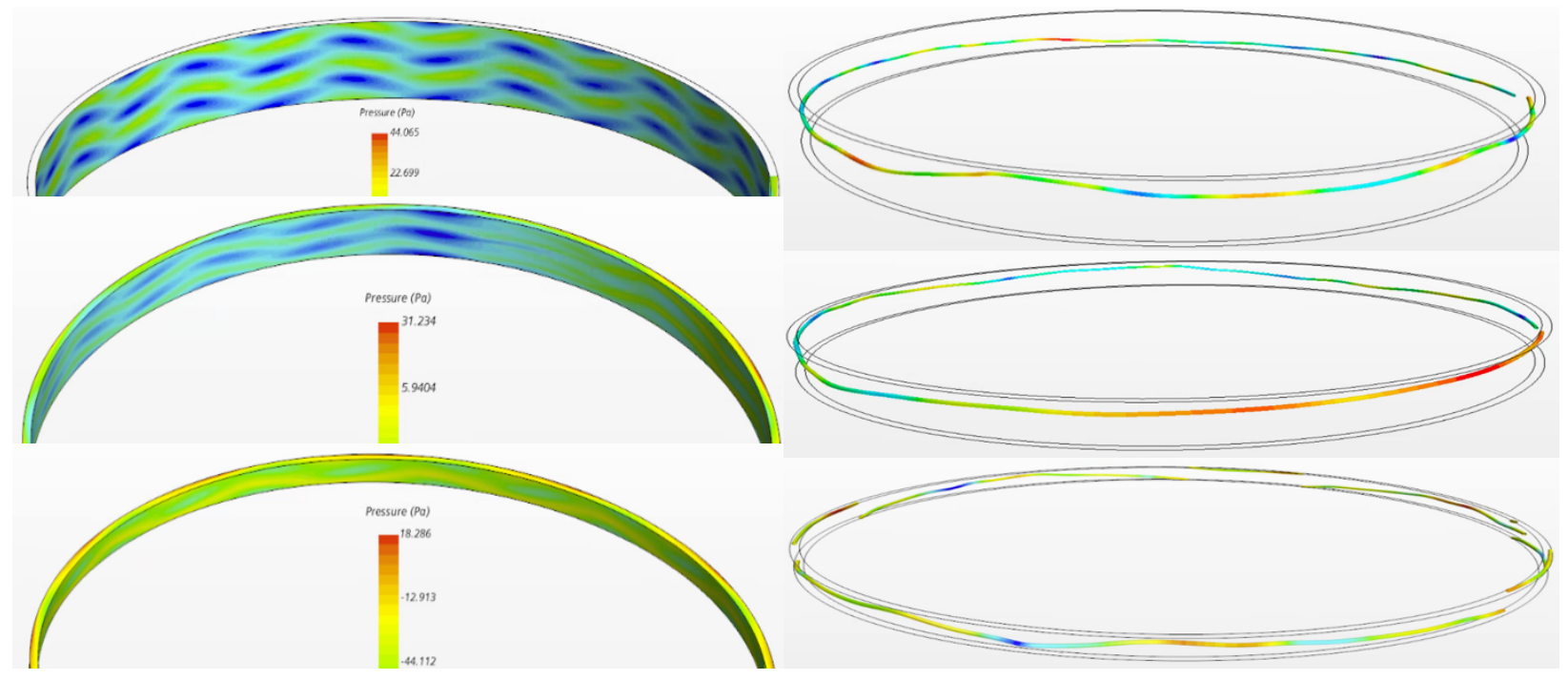

Figure 21. Vortex pattern as pressure imprint on the inner (rotating) cylinder wall (left) and particle tracks for one revolution (right) for the three domains 3,6 , and $9 \mathrm{~d}$ (from bottom up).

\subsection{ROTATIONAL SPEEDS CLOSE TO RATED (1,200-2,400 RPM)}

With an increasing inner cylinder speed, the flow structure did not change. The vortex pattern was preserved, as well as the vortex waviness. Figure 22 shows the results for three rotational speeds: 1,200, 1,800 , and 2,400 rpm, as computed with the $6 \mathrm{~d}$ model. Both $x$ and $y$ force components are plotted, and insets show the pressure on the inner wall, the particle track, and the vortex shedding. The results are quite similar to the those obtained at lower speeds. Except for sporadic spikes in force evolution, likely caused by numerical instability, the force remained low, with a magnitude not dependent on the speed. The force fluctuated at a frequency that corresponds to half of the cylinder's rotational speed, as noted above. The same tendency remained for up to the maximum speed of 2,400 rpm. It can also be concluded that these forces were caused by the rotating vortices and should not represent a factor in the magnetic bearing control because of their low intensity.

The vortex pattern remained unchanged with increasing speed. Two pairs of vortices are noticeable that do not travel in axial direction. When a particle track is computed, it shows that a single released particle stays almost confined within the $6 \mathrm{~d}$ space. A close observation of the circular vortex wavelength indicates a slight vortex stretching and widening as the speed increases. Twelve circular waves were counted for the 1,200 rpm case, whereas only 10 circular waves were counted for the 2,400 rpm speed. This finding is not significant and does not affect the overall impression regarding a preserved flow structure as the speed reaches maximum values. 

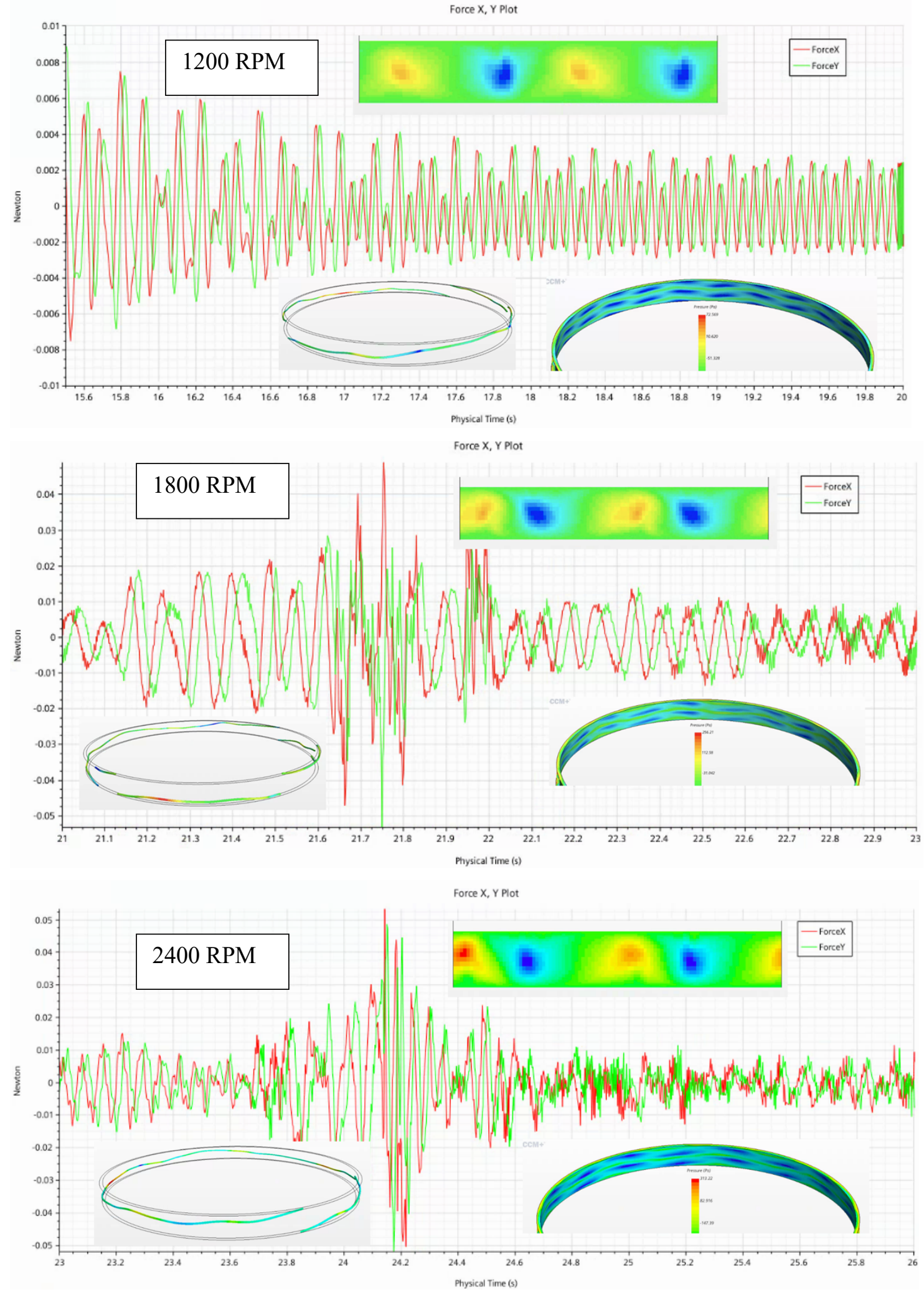

Figure 22. Combined plots of force, pressure imprint of the inner cylinder, particle track, and transverse gap velocity (vortex pattern) for rotational speeds of 1,200 (top), 1,800 (middle) and 2,400 (bottom) rpm. Computations were performed with the $6 \mathrm{~d}$ model. 
To exclude the possibility for an effect of the domain size, speeds of 1,200 and 1,500 rpm were computed with the $3 \mathrm{~d}$ model. The results are presented in Figure 23 and are similar to the results from the $6 \mathrm{~d}$ model. They are practically identical, except that the force had a lower magnitude because the model domain was twice as small. The force also oscillated at a frequency corresponding to the rotational speed. The oscillation magnitude varied, but it was still subject to sporadic spikes which were attributed to the numerical stability.
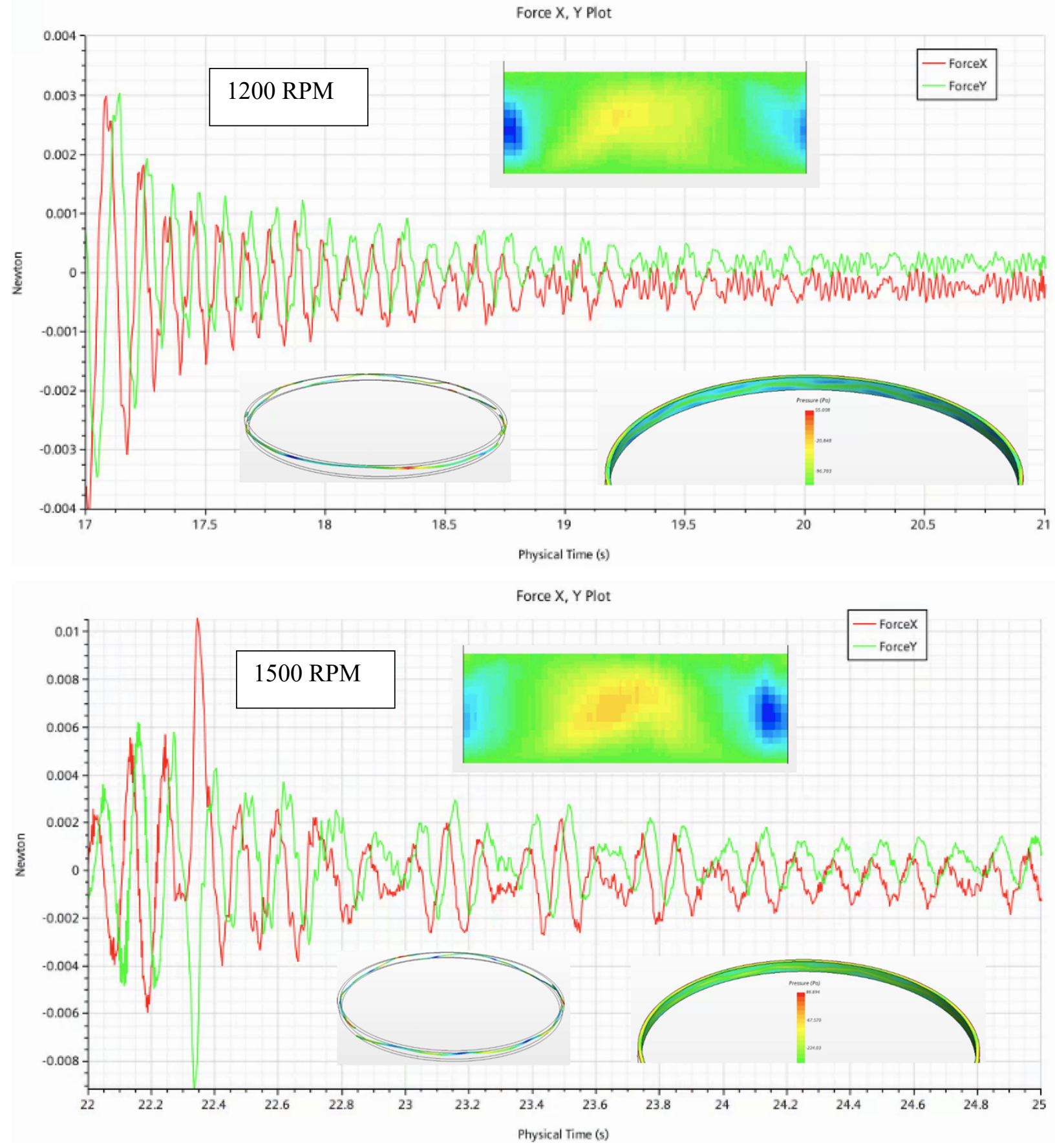

Figure 23. Combined plots from $3 \mathrm{~d}$ model calculations at rotational speeds of 1,200 and 1,500 rpm. Insets in the plots show the vortex imprint on the inner wall (lower right), the particle track (lower left), and the vortex shedding (top). 
With this scope of simulations, the concentric problem is considered sufficiently analyzed with the employed computational methods. When the cylinders are concentric, the generated fluid forces result from the vortex flow structure of the gap. Vortices transport fluid in the gap, thus equalizing the pressure gradient. At low speeds corresponding to the Couette regime, the flow has no vortical structure because the viscous forces damp the pressure gradient. As the rotational speed increases, the vortices appear. They have regular structure only in the Taylor regime, which was found to exist in a narrow speed range. For higher speeds, the vortices have a wavy structure which is preserved up to the maximum simulated speed of 2,400 rpm. The vortices generate fluctuating forces with low intensity, which should not present a concern for the magnetic bearing's controls.

\subsection{SUMMARY OF CONCLUSIONS FOR THE CONCENTRIC PROBLEM}

1. The force is very small and is caused by rotating vortices. In the ideal case with an adequately long cylinder ( $d$ in the order of hundreds), the force might be fully balanced and sum to zero.

2. Vortices forming in the gap have a wavy structure and are carried by the flow, with an average fluid speed of about half of the rotating cylinder speed. Their rotation causes force oscillations with the same frequency, for example, at a cylinder speed of $10 \mathrm{~Hz}$, the force oscillates with a frequency of $5 \mathrm{~Hz}$.

3. The force magnitude does not depend on the computational axial domain size. The observed differences likely have numerical origin (solution stability based on the time step or the inner iterations number), because the spatial discretization is kept the same.

4. The azimuthal vortex wavelength decreases according to the speed. The vortices stretch.

5. The vortices do not travel in an axial direction. Some deviation from a fully steady position is noticed, but this is confined within a $6 \mathrm{~d}$ axial space. 


\title{
5. ANALOGY TO THE LUBRICATION THEORY
}

An important question for salt pump magnetic bearing control is whether the lubrication theory can be applied, and to what extent, for analysis of the fluid's impact on the pump rotor. The lubrication theory provides a solid ground, with relatively low computational cost. The lubrication theory (tribology) is based on solutions of the Reynolds equation with a series of simplifications. The general form of the Reynolds equation in cylindrical coordinates can be found in multiple references. Below is a derivation given by Kirk (R. Kirk, 1975):

$$
\frac{1}{6}\left[\frac{1}{R^{2}} \frac{\partial}{\partial \theta}\left(\frac{h^{3}}{\mu} \frac{\partial P}{\partial \theta}\right)+\frac{\partial}{\partial z}\left(\frac{h^{3}}{\mu} \frac{\partial P}{\partial z}\right)\right]=\omega_{b} \frac{\partial h}{\partial \theta}+2 \frac{\partial h}{\partial t}
$$

where

\author{
$P$ is the pressure, \\ $h$ is the film thickness, \\ $R$ is the journal radius, \\ $\omega$ is the angular velocity, and \\ $\theta$ and $z$ are azimuthal and axial coordinates.
}

Two major simplifying assumptions are typically made: either the axial pressure derivative is zeroed, resulting in a "long" bearing, or the circumferential derivative is zeroed, resulting in a "short" bearing. Full analytical Reynolds equation solutions can be found in the literature (D. Sfyris, 2012).

The developed CFD model uses periodic axial boundary conditions, which make it a long bearing model analog. In tribology, a long bearing is a bearing with $\mathrm{L} / \mathrm{D}>>1$, where $\mathrm{L}$ is the length, and $\mathrm{D}$ is the diameter of the bearing. The rotor of the salt pump resembles a long bearing, with $L / D=230 / 66=\sim 3.5$

There are two basic properties that distinguish the pump rotor from a bearing: the gap, which compared to a typical bearing clearance is about 20 times larger, and the fluid viscosity, which is about the same magnitude lower. CFD simulations were used as described below to analyze similarities and to derive conclusions for potential use of lubrication practices for salt pump rotor performance.

\subsection{STATIC LOADS OF INFINITE BEARINGS}

The loads on rotating concentric bodies (rotor and stator) are dynamic when the rotor (or bearing journal) displacement is time dependent, and they are static when the displacement is constant. There are no forces (except those caused by vortices, as discussed in Section 0 ) if the bodies are concentric. In the Reynolds equation, the dynamic forces are expressed by the existence of film thickness $(h)$ temporal derivative. A comparison of numerical solutions for static forces against steady solution of Reynolds equation for long bearings was performed, as detailed in this section.

Sommerfield succeeded in solving the steady Reynolds equation for an infinitely long bearing. This solution is provided in many lubrication textbooks (O. Pinkus, 1961). First, the Reynolds equation was solved to obtain the pressure evolution, and then the loads were computed by integrating the pressure over the journal bearing surface. The bearing geometry, rotational frequency, loads, and lubricant viscosity can be combined in a nondimensional number named after Sommerfield (S), given by the following expression: 


$$
S=\frac{\mu N L D}{W}\left(\frac{R}{c}\right)^{2}
$$

where

$W$ is the load,

$N$ is the journal rotational frequency, and

$c$ is the clearance (gap).

This nondimensional number for long bearing static loading depends only on the relative clearance $\varepsilon=$ $d / c$, where $d$ is the journal displacement. It is given by the following relation:

$$
S=\frac{\left(2+\varepsilon^{2}\right)\left(1-\varepsilon^{2}\right)^{1 / 2}}{12 \pi^{2} \varepsilon}
$$

The relation given in Eq. (9) was obtained by allowing the gap pressure to become negative. It must be noted that in the simulations, the pressure also takes negative values, and it physically corresponds to the solution given above. Pinkus (O. Pinkus, 1961) provides an additional solution by modifying the pressure boundary conditions to limit the pressure within the positive range, which leads to a more complex form of the Sommerfeld number not shown here. Both numbers are plotted in Figure 24, with the negative pressure solution indicated by the solid green line, and the positive pressure solution indicated by the red dots.

To simulate the static forces on the pump rotor, a series of calculations was carried out by implementing stepwise increases in the displacement of the inner cylinder. After each step, the calculation was run long enough to allow the forces to stabilize and reach steady values. Figure 24 shows the result, as indicated by the black line. The numerical results are for two rotational speeds: $300 \mathrm{rpm}(5 \mathrm{~Hz})$ and $900 \mathrm{rpm}(15 \mathrm{~Hz})$. 

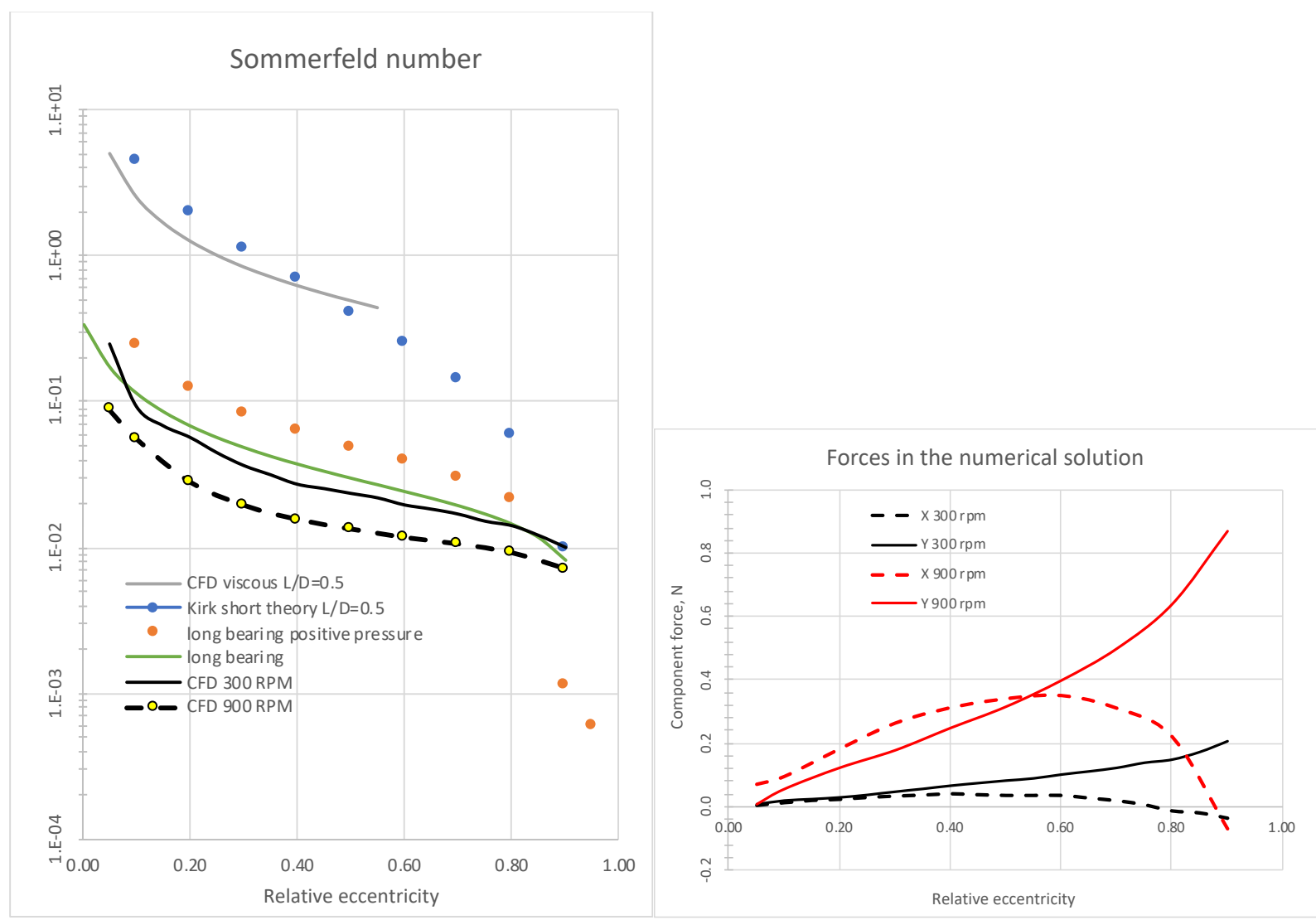

Figure 24. The plot on the left shows the Sommerfield (S) number calculation for the pump rotor and a comparison to theoretical solution for infinitely long bearings (left), accounting for the negative pressure (green line), and positive pressure (red markers) (O. Pinkus, 1961). The plot on the right shows component forces in the simulation.

The good agreement between the numerical and theoretical solutions indicates that the pump rotor static loads can be reasonably well predicted by a solution of the Reynolds equation for long bearings. The numerical result yields negative pressure, which produces higher loads compared to the $S$ number for positive pressure (red dots). The question about the physical meaning of the assumption for positive pressure requires further investigation. As the speed increases, the deviation from the theoretical $S$ number becomes larger: the ratio of frequency over the load is not constant. This can be explained by the growth of inertia effects, but no deeper analysis was carried out to determine the discrepancy's origin.

The left plot in Figure 24 also shows a different, perfunctory, yet valid numerical result (gray line) compared to a short bearing result from Kirk (R. Kirk, 1975). To achieve this solution, the viscosity in the model was increased about 7,500 times to match a typical $\mu / c^{2}$ term using data from Faria (M. Faria, 2014), and the clearance was left as it is for the salt pump. The calculations were not carried to high eccentricities, because the solution diverged. This result demonstrates the difference between the short and long bearing theories ( $S$ number slope) and the independence of $S$ from the fluid properties.

The numerical solution allows the component forces to be analyzed. The assumed displacement in the simulations was positive in the $x$ direction (load direction). When decomposed on $x$ and $y$ coordinates, the resultant reaction varies as the eccentricity increases (Figure 24, right). At small displacements, the reaction is pointed at almost 45 degrees relative to displacement direction ( $x$ and $y$ forces have almost the same magnitude), but with the gap closing, the reaction becomes more orthogonal to the displacement (load). The gap grows as the displacement increases. The force in line with the load (x-force) is positive for most of the $\varepsilon$ range, but at high eccentricities, it becomes negative. 
To further illustrate the differences between the numerical and the theoretical (Reynolds) solutions, the gap pressure is plotted in Figure 25. The major difference is seen in the pressure slope. The increased slope in the numerical solution causes a larger $Y$ component force. The discrepancy grows with higher rotational speeds, as seen in the left plot of Figure 24.

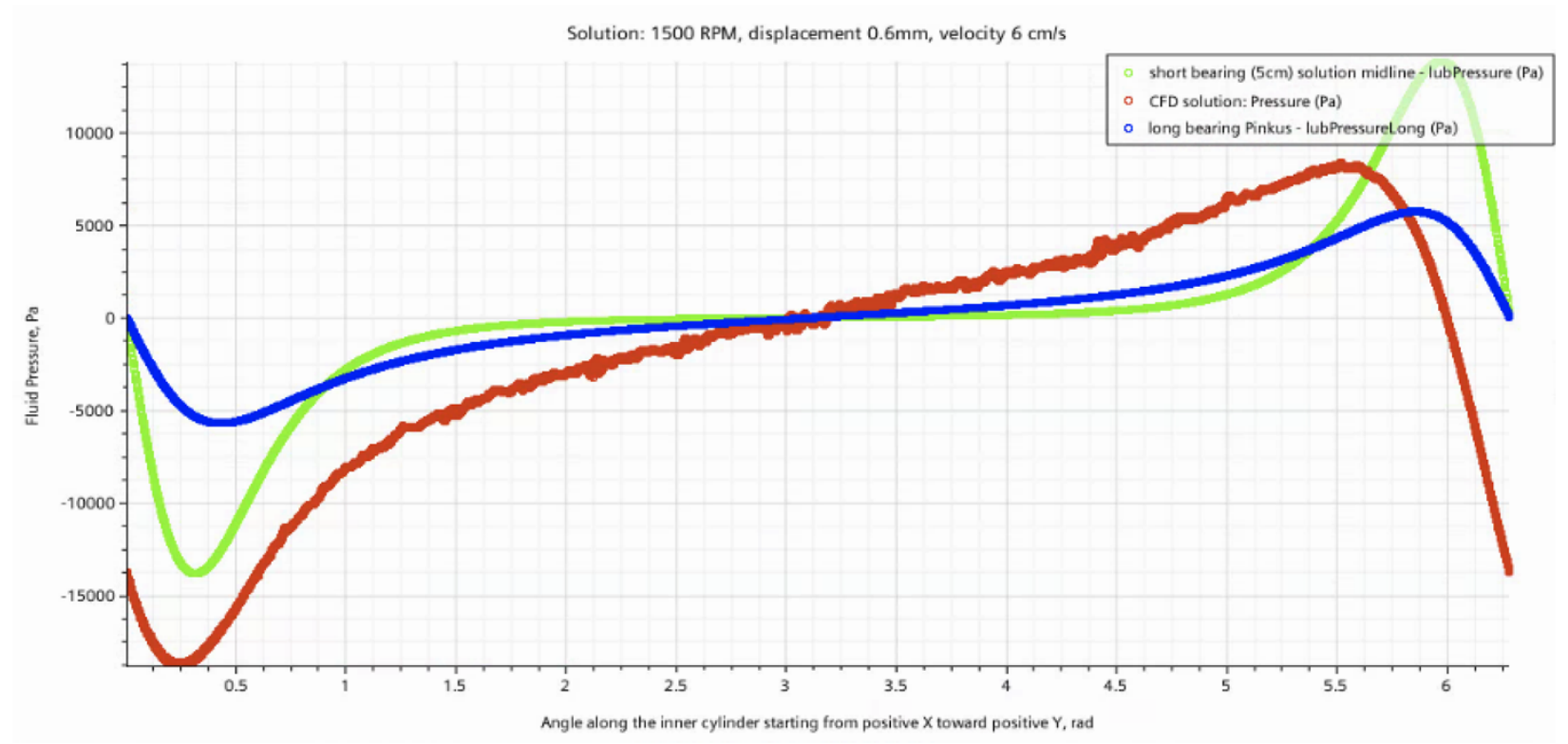

Figure 25. Static loading pressures from numerical solution (red), long bearing (blue), and short bearing (green).

\subsection{ANALOGY DURING DYNAMIC LOADING}

When the loading is dynamic, as when, due to an external force, the journal (rotor) moves, the forces are generally higher and different from the static forces. These forces are computed by solving the unsteady Reynolds equation. Again, two approximations are routinely used to simplify the solutions-long and short bearing approximations, as discussed above for the static problem. The boundary conditions are notably identical: for short bearings, the pressure is assumed to be equal to ambient (or zero) at the bearing extremities,

$$
\mathrm{P}(0)=\mathrm{P}(\mathrm{L})=0
$$

where $\mathrm{L}$ is the bearing length.

For long bearings, the pressure is zero at one position around the circumference:

$$
\mathrm{P}(0)=\mathrm{P}(2 \pi)=0
$$

For short bearings, the following solution for the pressure was given by Kirk (R. Kirk, 1975): 


$$
P(\theta, z)=-\frac{3 \mu z(z-L)}{4 h^{3}}\left[\omega \frac{\partial h}{\partial \theta}+2 \frac{\partial h}{\partial t}\right]
$$

The film thickness $h$ can be calculated by the relationship given below:

$$
h=c-x \cos (\theta)-y \sin (\theta)
$$

where

$h$ is the film thickness,

$c$ is the clearance,

$x$ and $y$ are the displacements, and

$\theta$ is the rotation angle relative to the center of bearing.

The derivation of this approximate relationship was not provided in Kirk (R. Kirk, 1975). An evaluation of the accuracy of this expression as applied to the salt pump geometry was carried out as described below, and the problem is illustrated in Figure 26. The displaced journal is shown with the dashed line: the displacement was only in the positive $x$ direction.

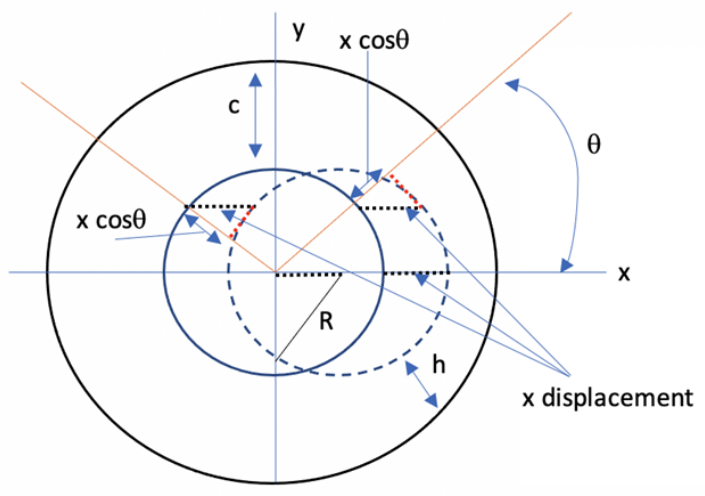

Figure 26. Film thickness calculation and error evaluation.

The $\mathrm{x}$ displacement is marked with the dotted black lines on the diagram. Four discrete angles were considered: zero, a positive cosine, 90 degrees, and a negative $\operatorname{cosine}$. The term $x \cos \theta$ is also indicated. The error in calculating the film thickness appears as a distance between the red dotted line and the dashed line of the displaced journal. The error is zero for angles of 0 and 180 degrees and is the largest for angles of 90 and 270 degrees, as seen in Figure 26. The calculated film thickness is always smaller than the real one. It is possible to evaluate the largest error at 90 degrees for the AMB geometry with the following expression:

$$
(R+c)-\sqrt{R^{2}-x^{2}}-c=33-\sqrt{33^{2}-0.5^{2}}=0.0038 \mathrm{~mm},
$$


where $R$ is the journal radius. Relative to the clearance $c(0.75 \mathrm{~mm})$, the error is $0.5 \%$. This maximum error is acceptable for the current application, and the above formula for the film thickness is used further in the analyses.

For long bearings, a solution can be found in the textbook of Pinkus (O. Pinkus, 1961) in the following form:

$$
P(\theta)=6 \mu\left(\frac{R}{c}\right)^{2}\left[\frac{(2+\varepsilon \cdot \cos \theta)}{(1+\varepsilon \cdot \cos \theta)^{2}} \omega \frac{(\varepsilon \cdot \sin \theta)}{2+\varepsilon^{2}}+\frac{1}{\varepsilon}\left(\frac{1}{(1+\varepsilon \cdot \cos \theta)^{2}}-\frac{1}{(1+\varepsilon)^{2}}\right) \frac{\partial \varepsilon}{\partial t}\right]
$$

where $\varepsilon=x / c$ is the relative eccentricity

The film thickness time derivative relates to the eccentricity time derivative, as follows:

$$
\frac{\partial h}{\partial t}=-c \frac{\partial \varepsilon}{\partial t} \cos \theta
$$

and they both can be computed knowing the displacement velocity $\dot{x}$ (grid velocity in simulation),

$$
\frac{\partial h}{\partial t}=-\dot{x} \cdot \cos \theta \quad \frac{\partial \varepsilon}{\partial t}=\frac{1}{c} \dot{x}
$$

The computations were performed using the following approach. First, the long and short bearing pressures were compared to the computed pressure. The forces were calculated based on these pressures using the integration algorithm explained above. Results are discussed below.

As in the static problem, the largest difference is seen in the $Y$ force component. The Reynolds solutions (both short and long) compute much smaller, positive $Y$ force. The $X$ force is properly predicted, resulting in a negative (against the motion) reaction (Figure 27). In the theoretical solutions, the $X$ force is dominant, whereas in the numerical result, the $Y$ force is dominant. 


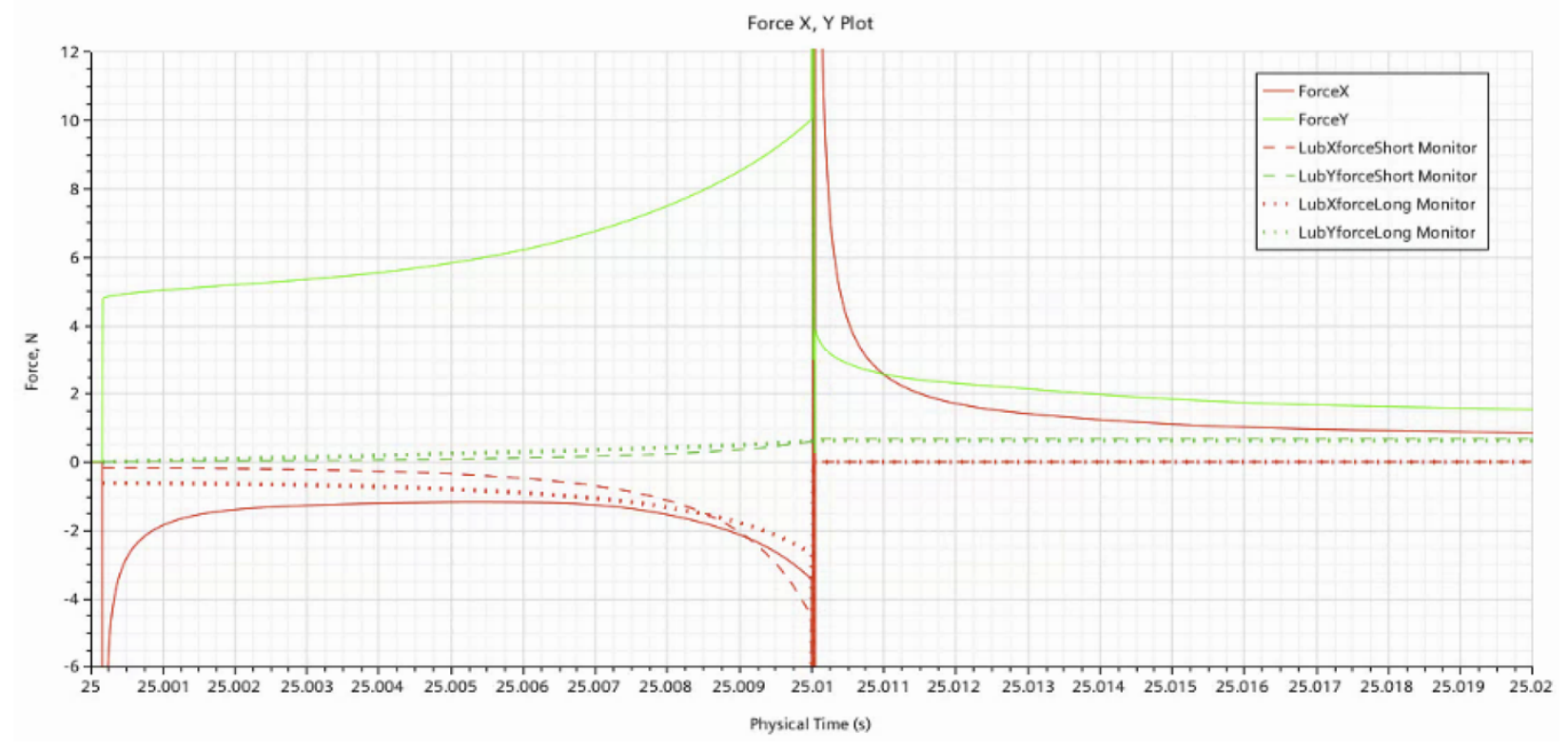

Figure 27. Forces during dynamic (25-25.01 s) and static (25.01-25.02) loading for 1500 rpm. Solid lines indicate CFD, dotted lines indicate long bearing, and dashed lines indicate short bearing.

The large $Y$ force appearance is explained by the existence of a pressure gradient in the gap (Figure 28) in the calculation. The computed pressure becomes negative, contrary to the Reynolds pressure, which stays positive. Pinkus (O. Pinkus, 1961) writes that for bearings, a negative pressure is not possible, thus affirming that the Reynolds equation should be solved with boundary conditions that will prevent the pressure from becoming negative. In the simulations, a periodic boundary condition was used on the sides in the axial direction. This approach results in an infinitely long configuration, and the liquid (lubricant) was restrained to remain in the gap. To verify the influence of the axial boundary condition on the pressure evolution in the gap, a finite configuration with $20 \mathrm{~d}$ length was solved under constant pressure boundary conditions that would allow incoming/outgoing liquid fluxes to exist (breathing). Results are presented below. 


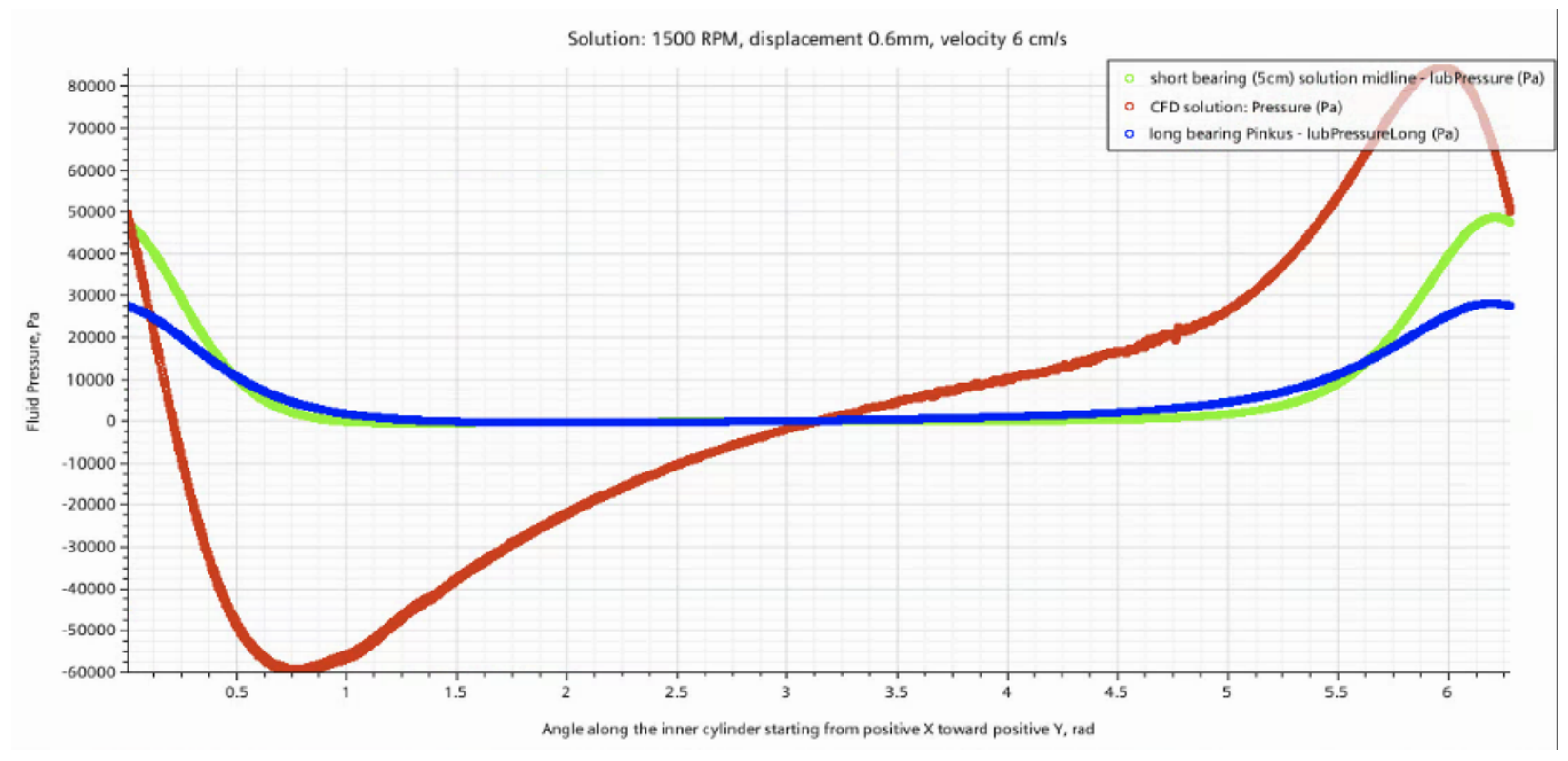

Figure 28. Loading pressures at the end of dynamic linear motion with $6 \mathrm{~cm} / \mathrm{s}$ from numerical the solution (red), long bearing (blue), and short bearing (green).

\subsection{POSITIVE GAP PRESSURE SOLUTION, DOMAIN 20 D}

Positive gap pressure indicates that the pressure in the gap during dynamic and static loading remains positive in the absolute sense (i.e., it can still be lower than the ambient). In the simulations, the ambient pressure was assumed to be zero for convenience, but often it can be assumed to be equal to the atmospheric pressure (1 bar) or even higher. In the case of zero ambient pressure, negative pressures with magnitudes within the ambient pressure still satisfy the "positive" pressure assumption.

A simulation was set up using the same computational methods, but with two modifications: the domain was increased to $20 \mathrm{~d}(15 \mathrm{~mm}, \mathrm{~L} / \mathrm{D} \sim 1 / 4)$, and zero pressure boundary conditions were imposed on the two axial extremities. The boundary conditions were open flow, which allowed the flow to enter and exit from the domain, resulting in some recirculation at the ends. Techniques were applied to minimize this recirculation and to achieve as much even pressure distribution as possible at the side surfaces (element faces). A simulation was run at $600 \mathrm{rpm}$ and $6 \mathrm{~cm} / \mathrm{s}$ linear displacement of $0.6 \mathrm{~mm}$, as before. Results were compared to short and long bearing pressures and forces.

The result of the constant pressure boundary condition is evident, leading to positive pressures in the gap. It is notably comparable to the lubrication pressures (Figure 29). The magnitude varies because the simulated configuration is finite and produces lower pressure than the long bearing Reynolds equation solution (blue line). The short bearing pressure (highest pressure) is overestimated because a finite length of $5 \mathrm{~cm}$ was assumed, which produces higher pressure. Figure 29 has only qualitative meaning, illustrating that the calculated and the theoretical pressures are alike. This observation is valid for both dynamic and static loading (top and bottom plots in Figure 29).

The resultant forces decomposed on the two principal axes are shown in Figure 30. As seen above in the periodic configuration (Figure 28), the large $Y$ force has disappeared, confirming the better analogy to the lubrication theory solution. The $Y$ force magnitudes are very close in both dynamic and static loading, except for the short bearing which is higher. The major reaction is on the $X$ axis and opposes the motion. The magnitudes are significantly different, as explained above. 

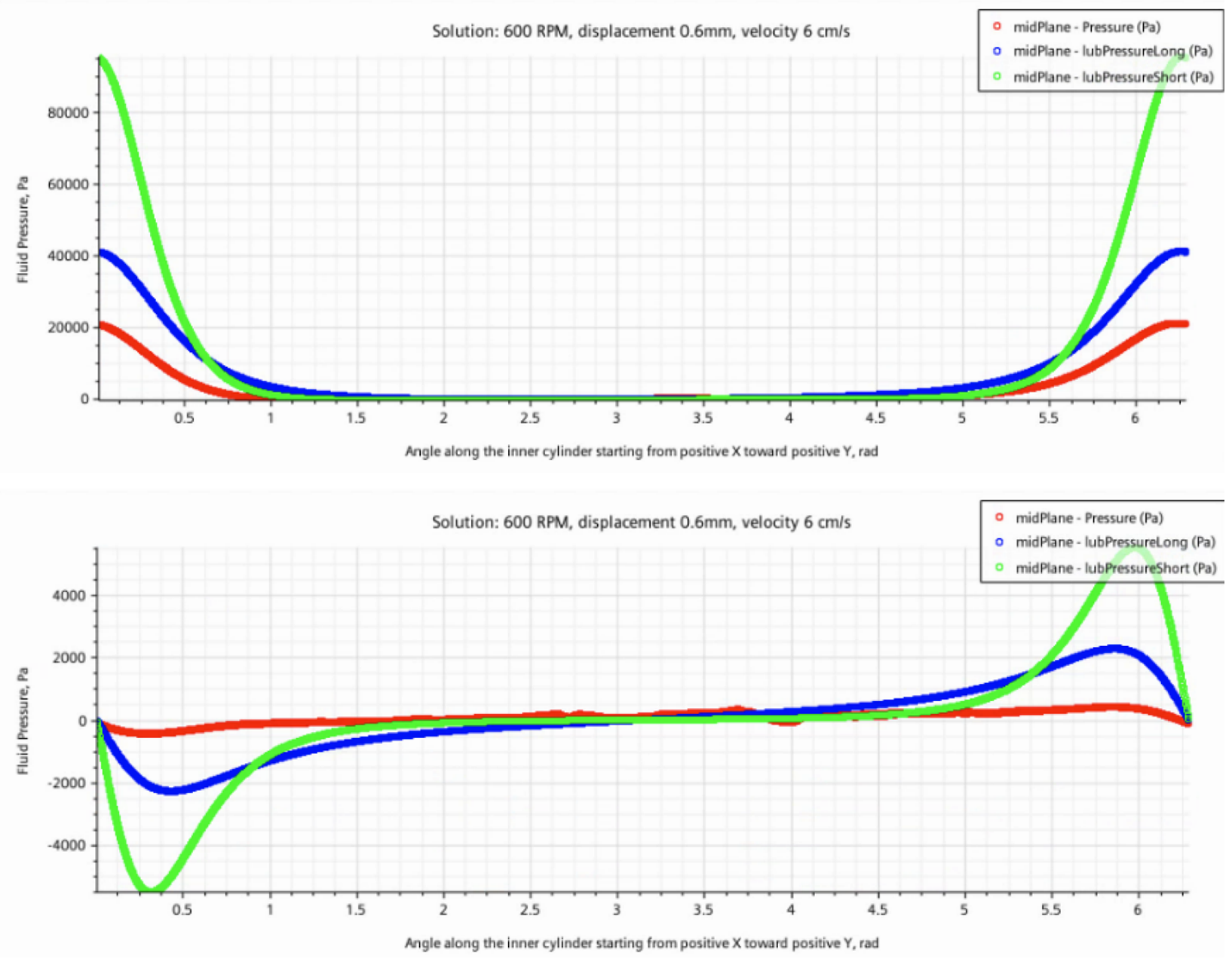

Figure 29. Midline gap pressure at the end of the linear motion (top), and during the static operation (bottom), for the three configurations: simulation (red), long bearing (blue), and short bearing (green).

Figure 31 illustrates the pressure contours on the rotor at the end of the calculated period during static conditions. The pressure is close to zero (side condition pressure) in the entire domain except in the minimal gap zone, where it transitions sharply from negative to positive. The same is also plotted in Figure 29. Although it is a finite length solution, because of the short domain simulated $(15 \mathrm{~mm})$, it resembles the short bearing lubrication solution more, where the azimuthal pressure derivative is zeroed. To evaluate whether the long or short theory is more appropriate for this application to the salt pump rotor, a full-size problem must be simulated. 


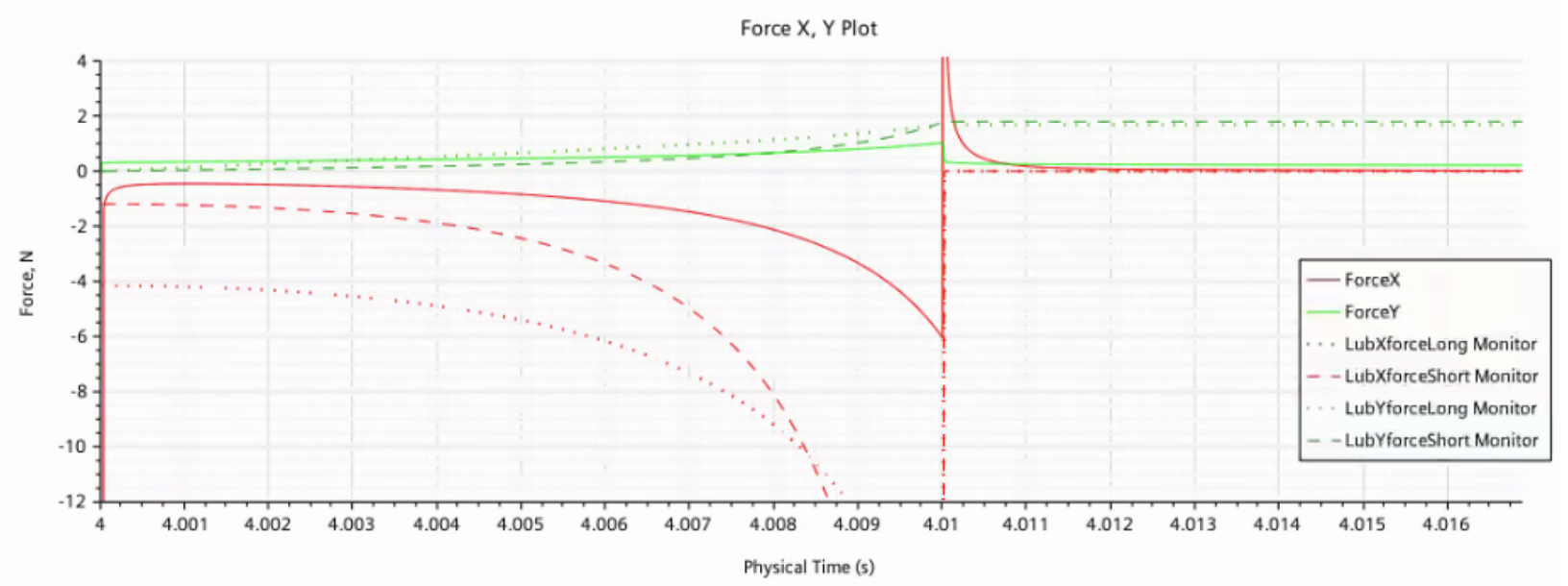

Figure 30. Forces during dynamic and static loading with constant pressure boundary conditions and finite rotor length (20 d) for simulation (solid line) and lubrication theory (dotted lines). $X$ component forces are shown with red, and $Y$ component forces are indicated with green.
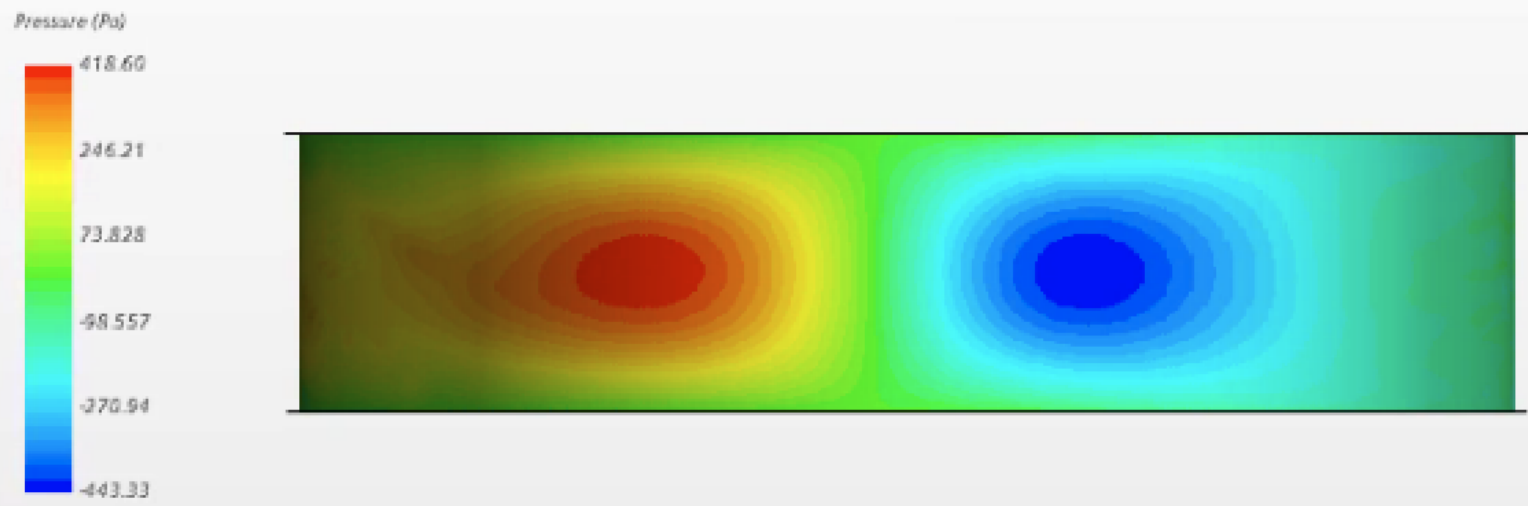

Figure 31. Gap pressure distribution in the simulation in the static regime in the vicinity of minimal gap. 


\section{FORCES IN ECCENTRIC CYLINDERS}

The computation of fluidic forces is discussed in the previous section. Here, a more comprehensive approach to the same problem is presented, with a focus on the extended operational range. The results are presented in a systematic form, readily portable to the bearing control algorithms.

The fluidic forces grow as the inner cylinder is offset. This is caused by pressure redistribution in the gap. The force magnitude and direction relative to the inner cylinder motion is of primary interest for magnetic bearing control. To properly model the motion of the inner cylinder, the computational models are amended with grid morphing functionality. Grid morphing is a computational technique that allows the grid to be physically changed at each time step to simulate a time-dependent motion. Two parameters define the grid motion: the displacement, and the grid velocity. The simulations were performed in the cartesian coordinate system, in which the displacement is decomposed in $x$ and $y$ directions, and the velocity is defined as the time derivatives of displacement in these two coordinates:

$$
\frac{\partial x}{\partial t}=\dot{x} \text { and } \frac{\partial y}{\partial t}=\dot{y}
$$

\subsection{LINEAR ROTOR MOTION, LONG BEARING ANALOG}

First, a linear motion of the rotor in the $X$ direction was simulated in periodic axial conditions (long bearing). The gap size $d$ (in some references, the clearance, $c$ ) of the current pump design is $d=0.75 \mathrm{~mm}$. To prevent a full gap closure and to accommodate a tolerable grid distortion during grid morphing, a maximum displacement of $0.6 \mathrm{~mm}$ was assumed, resulting in a minimum gap of $0.15 \mathrm{~mm}$ and a relative eccentricity $\varepsilon=0.6 / 0.75=0.8$. A linear velocity of $6 \mathrm{~cm} / \mathrm{s}$ at five rotational speeds $-300,900,1,200$, 1,500 and $1,800 \mathrm{rpm}$ was deemed to be representative for the rotor motion in the real configuration. The time advancement was performed with a timestep selected based on two factors: (1) at least 500 time steps would occur during the motion period, and (2) a minimum of 3,000 time steps would occur within a full cylinder rotation. The data used in the simulations are given in Table 5.

Table 5. Simulation input data for linear motion of inner cylinder in the $X$ direction with a maximum displacement of $0.6 \mathrm{~mm}$

\begin{tabular}{|c|c|c|c|c|c|c|}
\hline $\begin{array}{l}\text { Velocity, } \\
\mathrm{m} / \mathrm{s}\end{array}$ & $\begin{array}{c}\text { Displacement } \\
\text { time (s) }\end{array}$ & $\begin{array}{c}\text { Time } \\
\text { step (s) }\end{array}$ & $\begin{array}{c}\text { Computed } \\
\text { physical time } \\
(\mathrm{s})\end{array}$ & $\begin{array}{c}\text { Rotational } \\
\text { speed (rpm) }\end{array}$ & $\begin{array}{c}\text { Inner cylinder } \\
\text { rotations }\end{array}$ & $\begin{array}{c}\text { Time steps per } \\
\text { revolution }\end{array}$ \\
\hline 0.06 & 0.01 & $2 \mathrm{e}-5$ & 0.02 & 300 & 0.1 & 10,000 \\
& & $2 \mathrm{e}-5$ & & 900 & 0.3 & 3,333 \\
& & $1 \mathrm{e}-5$ & & 1,200 & 0.4 & 5,000 \\
& & $1 \mathrm{e}-5$ & & 1,500 & 0.5 & 4,000 \\
& & $1 \mathrm{e}-5$ & & 1,800 & 0.6 & 3,333 \\
\hline
\end{tabular}

The forces per unit length are plotted in Figure 32. A period twice as long was computed to allow for simulation of the dynamic and static responses. Figure 32 shows the force components. During the dynamic period $(0-0.01 \mathrm{~s})$, the $\mathrm{X}$ force is negative, opposing the motion, and the $Y$ force is positive, resulting in a reaction offset in the direction of rotation. As the gap closes, the forces increase, with the highest force occurring at the smallest gap. After the motion stops at $0.01 \mathrm{~s}$, the force magnitude 
decreases, and the $X$ component changes from negative to positive. The rotation speed has strong influence only on the $Y$ component force, which grows in proportion to the speed. The $X$ component is not largely affected. This force response to the rotational frequency could be attributed to the inertial effects of the moving liquid. The boundary conditions corresponding to a long bearing also have a significant effect on the force, as pressure in the gap can assume negative values. The static forces are also affected by the speed of rotation, and they grow as the speed increases.

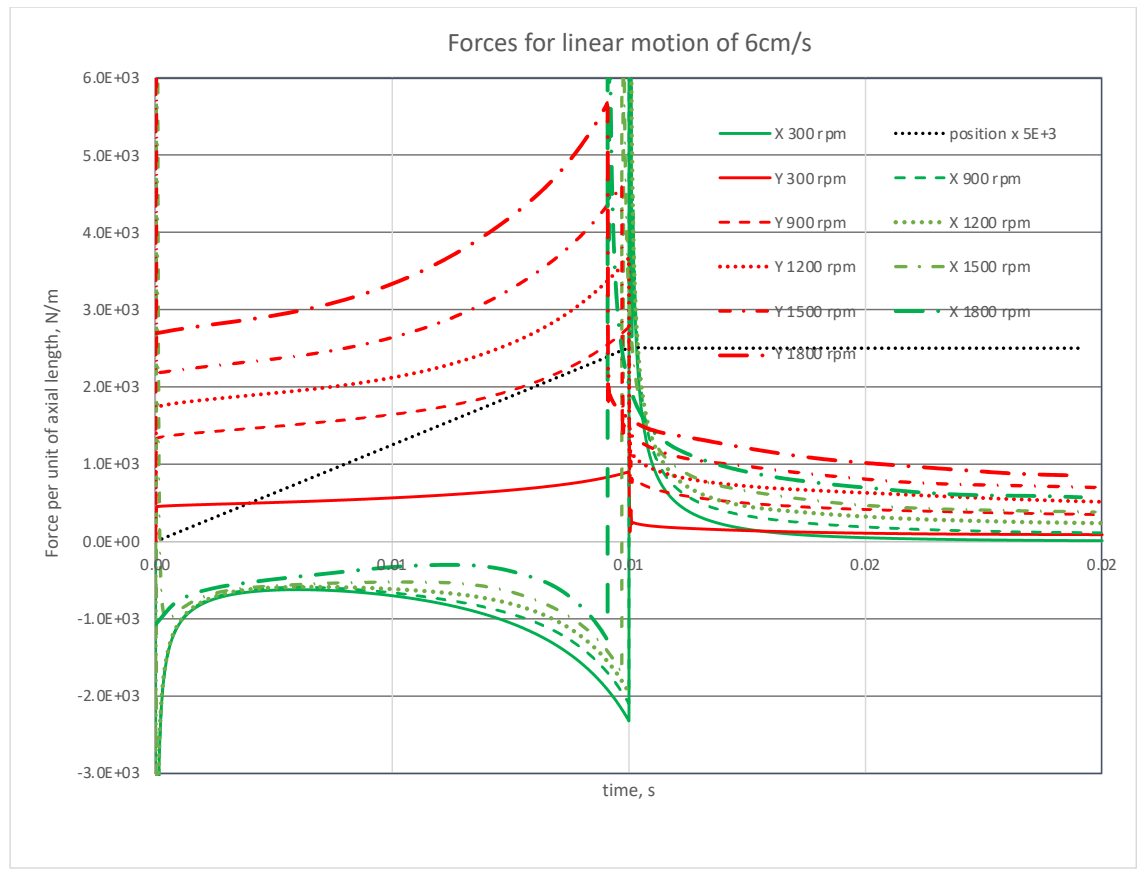

Figure 32. Dynamic and static forces during and after a linear motion of rotor (journal) of $6 \mathrm{~cm} / \mathrm{s} \mathrm{and} \mathrm{a}$ maximum relative eccentricity $\varepsilon=0.8$. Dynamic forces are in the $0-0.01 \mathrm{~s}$ time interval, and static forces are in the 0.01-0.02 s time interval. The relative displacement is indicated by black dots.

\subsection{SINE MOTION OF THE INNER CYLINDER, POSITIVE PRESSURE ANALOG, REAL BEARING}

The model that uses constant pressure boundary conditions at the two axial extremities of the rotor (see Section 5.3) was used to perform this simulation. Two cycles of sine motion along the $X$ axis, each with a frequency of $31.83 \mathrm{~Hz}$, were simulated. The plot on the left in Figure 33 shows the forces, and the motion (position) is plotted on the right. The forces change direction as the position changes direction, following a periodic evolution. The $X$ force opposes the motion: during the positive $X$ motion, the force is negative, and vice versa. The force is characterized with a two-hump structure because when the gap is small (humps), the force is the greatest. The hump profile is well represented in the simulations and is equally represented in the lubrication forces. It is perfectly symmetric in the lubrication solution, but in the simulation, the first hump is larger. Another peculiarity is that the simulation has a time shift of about 2 milliseconds for the selected set of frequency and rotational speed from the zero-velocity point. The lubrication forces change direction exactly at the zero-velocity point, whereas the simulated force changes early. This indicates that during this time interval, the force tends to close the gap. This finding may require further analysis. 

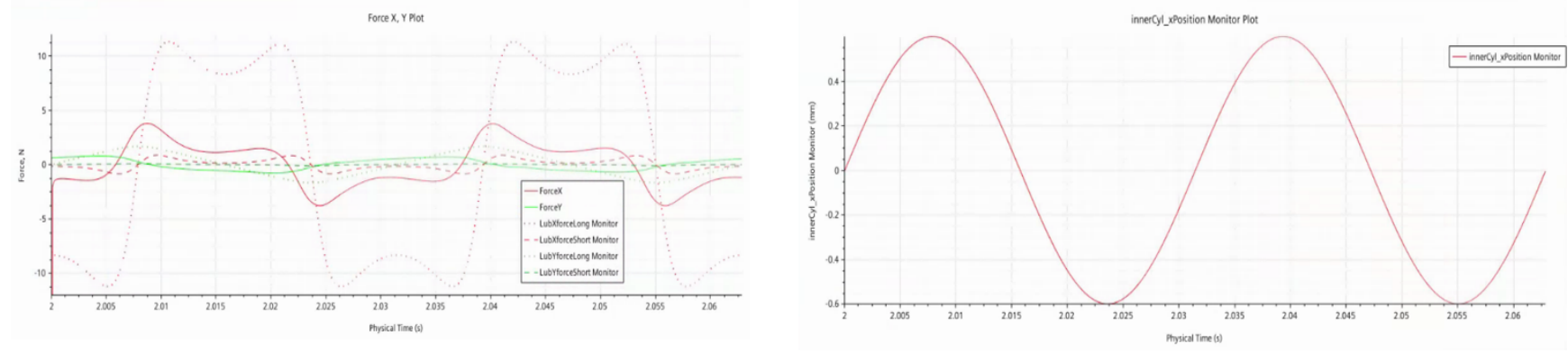

Figure 33. Forces (left) and rotor position (right) during sine motion with a frequency of $31.83 \mathrm{~Hz}$; two cycles modeled.

Figure 34 illustrates the pressure distribution in the gap. The left side shows the high-pressure spot on the inner cylinder wall in the simulation. The time instance corresponds to the highest velocity period of motion in positive (top) and negative (bottom) directions. The plots on the right show the mid-gap pressure distribution in the simulation and as calculated for the lubrication pressures (long and short). The shift of 180 degrees is clearly seen. The magnitude is identical for the positive and negative motions. The long bearing solution pressure is negative in the negative motion, which might be a numerical artifact. However, it produces the correct force with regard to time and direction.
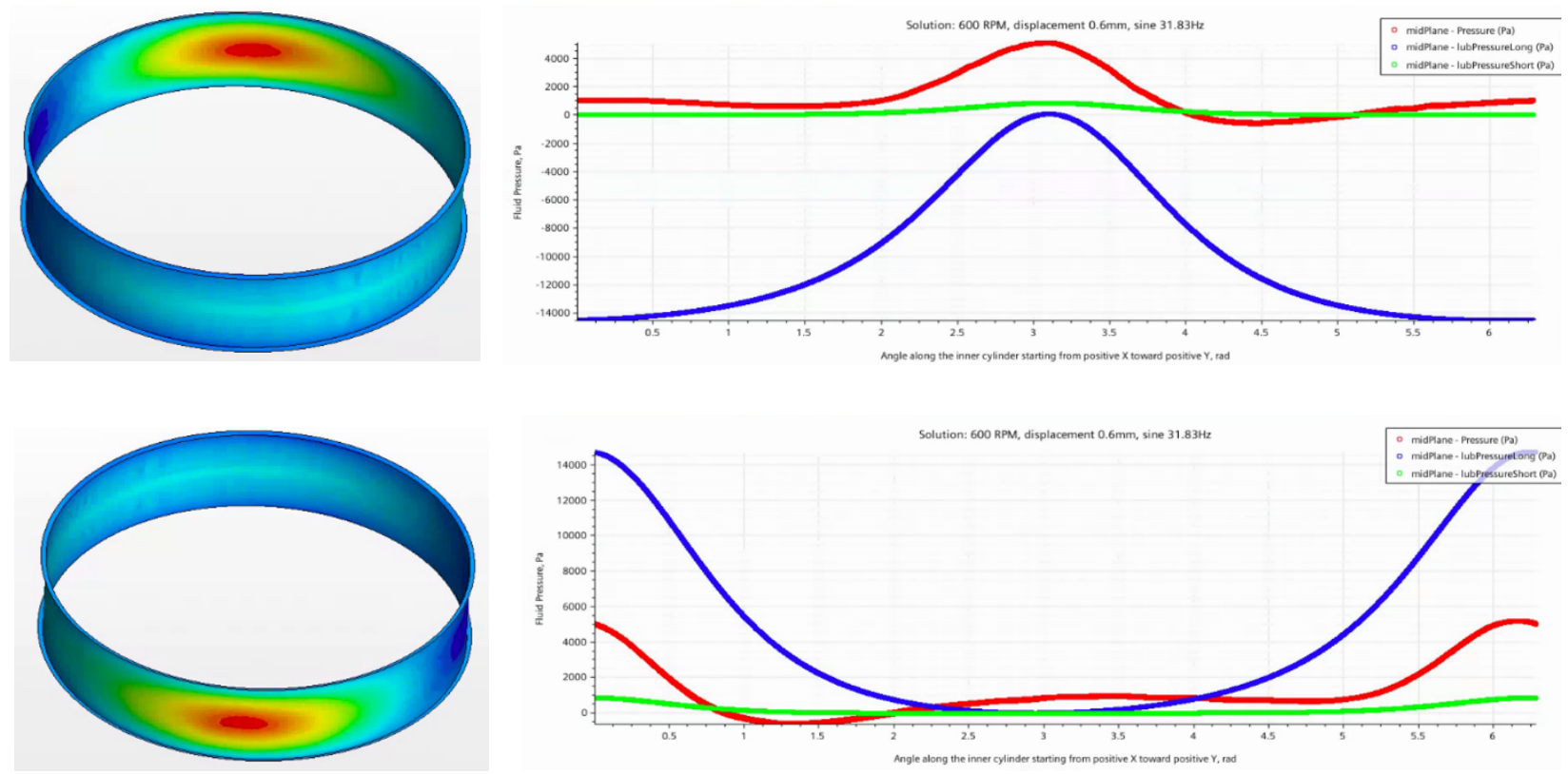

Figure 34. Pressures during sine motion. The pressure imprint on the inner cylinder wall is shown on the left, and the midpoint pressure distribution along the circumference for simulation pressure (red), long bearing (blue), and short bearing (green) are plotted on the right. The top plots show pressures during a positive cycle, and the lower plots show a negative cycle at a point of almost minimal gap, just before the velocity becomes zero.

\subsection{ROTATIONAL SPEED IMPACT ON PHASE SHIFT AND FORCE MAGNITUDE}

To understand the origin of phase shift in the simulation, more rotational speeds (1,200 and 1,800 rpm) were computed. The evaluation was focused on two phenomena: (1) the shift in time (phase) between the computed and lubrication forces at the point when the rotor stops in the minimum gap position (when the $X$ force component becomes zero and the $Y$ component is at a maximum), and (2) the asymmetry in the $X$ 
force magnitude (humps) when the gap opens (larger hump) and when it closes (smaller hump). Results are discussed below.
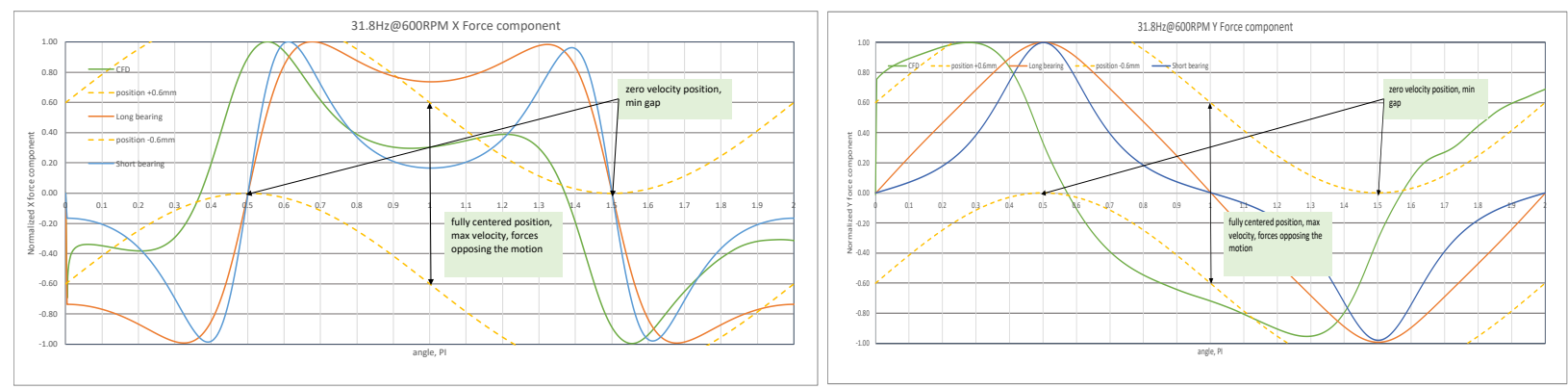

Figure 35. Phase shift evaluation plot with normalized $X$ and $Y$ forces from simulation. Long and short bearings are plotted against the rotor position bounds to allow for computation of the phase shift.

Figure 35 depicts the phase shift. The forces are normalized to eliminate the magnitude effect. The sine motion position plot is offset in both positive and negative directions, as indicated by the dashed position lines, to illustrate the minimum gap (zero velocity) point on the horizontal axis. The lubrication $X$ force (short and long) becomes zero, and the $Y$ force becomes maximum at the exact same point, whereas in the simulation, the $X$ force zero-point experiences a phase shift of 0.12 PI ( 2 milliseconds), and the $Y$ force maximum point shift is $0.2 \mathrm{PI}$ ( 3 milliseconds) preceding the zero-velocity point. Therefore, during this period, the $X$ force is not opposing the motion, but is instead facilitating the gap closure. The shift is invariant from the motion direction. The lubrication $X$ forces do not demonstrate such behavior. They change direction at the zero-velocity point.

Analyzing the result, the following observations can be made:

1. The $X$ force always opposes the motion. It is negative when the motion is positive, and vice versa, assuming the direction (positive or negative) is determined by the axis orientation.

2. The first observation above is true regardless of the gap opening or closing: the force opposes the opening of one of the gaps, and then it opposes the opposite gap to close. This is likely a result of the imposed continuous rotor motion.

3. When the position is centered, according to previous concentric analyses, no forces should develop. However, at that point, the motion velocity is the highest, and the force is still opposing the motion, but it has lower magnitude, so it forms a humped profile. This is purely a dynamic effect. The $Y$ component at that point is zero, meaning the force is aligned (against) with the motion. The same is not true for the computed $Y$ force, which has a strong magnitude at the centered position.

4. The $Y$ force changes its sign at the centered position and zeroes down. It is at its maximum at the minimum gap position (zero velocity). The $Y$ force has a sine-like shape in phase with the motion. The short bearing force shows inflation contrary to the long bearing, which could result from the final length of the bearing.

5. The computed $Y$ force is also periodic, with some inflation that follows the motion frequency with some phase shift, as explained above. The distortion reduces the $Y$ force magnitude at the minimum gap position, thus decreasing the likelihood of gap closure from fluid forces. 

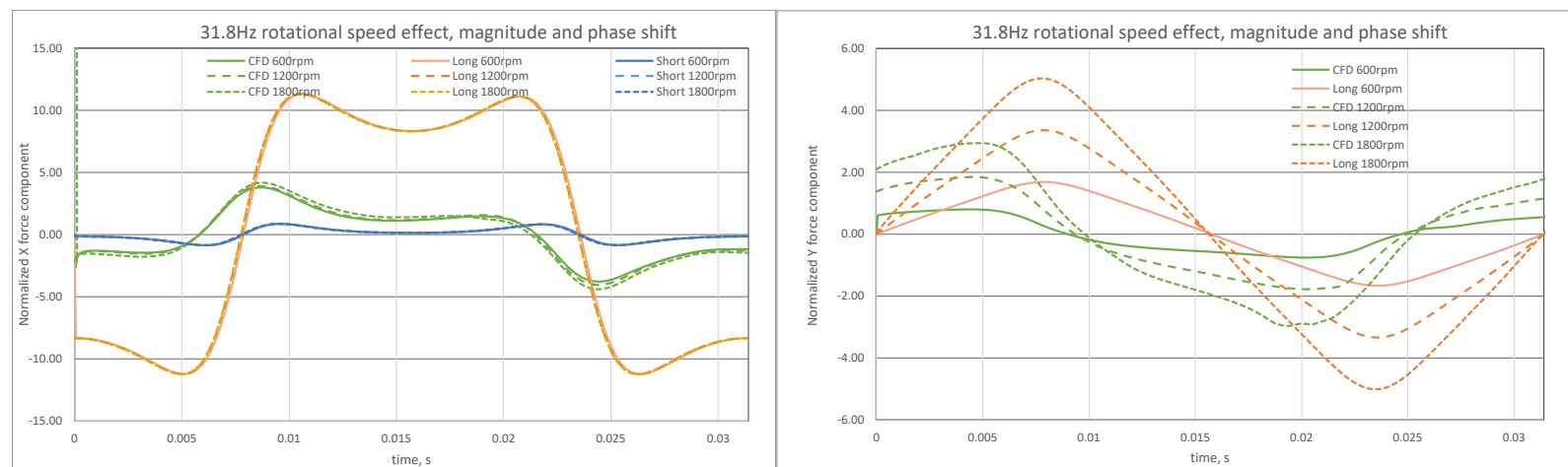

Figure 36. Rotational speed effect. $X$ (left) and $Y$ (right) forces resulting from $31.8 \mathrm{~Hz}$ sine motion are plotted for three rotational speeds: $600,1,200$, and $1,800 \mathrm{rpm}$.

Figure 36 shows the rotational speed effect on the force magnitude and phase shift. The forces are plotted in the same scale to permit the comparison. The $X$ forces fully coincide for all three speeds, so the speed of rotation does not affect the force magnitude or the phase shift. The same is not true for the $Y$ force component, in which the force grows proportionally to the speed. Furthermore, the $Y$ force shift is not affected, either. 


\section{CONCLUSIONS}

CFD analyses of forces developing between the rotor and stator of an immersed in molten salt pump were carried out. The obtained results (force magnitudes and directions) can be used to inform a solid body dynamic model of a pump rotor for the purposes of magnetic bearing control. The numerical analyses can be grouped into three categories:

- Static analyses of concentric configurations. A range of speeds was analyzed, ranging from purely viscous conditions up to high rotational speeds. These studies demonstrated that the developed models successfully replicate the well-known flow regimes (Couette, Taylor) and were thus validated. It was also concluded that when the rotor-stator configuration is perfectly concentric, the observed forces are caused by rotating wavy vortices and have low magnitude, which is insignificant for the bearing operation.

- Analyses of rotor static loading. Static loadings were analyzed under the assumption that negative pressure can develop in the gap. Along the computed forces, solutions of the Reynolds equation for long and short bearings were carried out to analyze the analogy to the lubrication theory. It was confirmed that the lubrication theory can be used if the rotor design allows the pressure to stay positive relative to the ambient pressure.

- Analyses of rotor dynamic loading. The dynamic loading is of primary interest for active magnetic bearing control. The response to periodic rotor motion was analyzed. The results were compared to the corresponding lubrication forces and show very good similarity, with two exceptions: a time shift between the computed and lubrication forces was observed, and a difference was seen between the axial (X) forces during the gap opening and closing.

The results suggest the CFD computations should be continued by implementing a rigid solid dynamics rotor model that will allow for closing the fluid and solid coupling of the problem. These computations will also permit further investigation into the extent that the lubrication idealized solutions are applicable to the real molten salt pump configuration. The developed modeling approach and experience gained can be used to create a digital analog (twin) of the pump/AMB for further study of the control logic.

\section{Acknowledgement:}

Funding for this was provided by the US Department of Energy Advanced Research Program Agency through the MEITNER program. The project was performed under the MEITNER Resource Team in support of the development of a magnetically suspended canned rotor pump for the integral molten salt reactor designs. 


\section{REFERENCES}

A. Melin, R. Kisner, D. Fugate. 2013. Embedded Sensors and Controls to Improve Component Performance and Reliability - Bench scale Testbed Design Report. s.1. : ORNL/TM-2015/XXX, 2013.

A. Recktenwald, M. Lucke, H. Muller. 1993. Taylor vortex formation in axial through flow: Linear and weakly nonlinear analysis. s.1. : Phis. Rev. E, 48,, 1993.

Calnetics, Technologies. 2020. https://www.calnetix.com/resource/magnetic-bearings/position-controlmagnetic-bearing. 2020.

D. Deng. 2007. A Numerical and Experimental Investigation of Tylor Flow Instabilities in Narrow Gaps and Their Relationship to Turbulent Flow in Bearings. s.l. : University of Akron, 2007.

D. Sfyris, A. Chasalevris. 2012. An exact analytical solution of the Reynolds equation for the finite journal bearing lubrication. s.l. : Tribology international 55, 2012.

M. Couette. 1890. Études sur le frottement des liquides. s.1. : Ann. Chim. Phys., 1890.

M. Faria. 2014. On the Hydrodynamic Long Journal Bearing Theory. London, July 2-4 : World Congress of Engineering vol II, 2014.

O. Pinkus, B. Sternlicht. 1961. Theory of hydrodynamic lubrication. s.1. : McGraw-Hill, 1961.

R. Kirk, E. Gunter. 1975. Short Bearing Analysis Applied to Rotor Dynamics. s.1. : ASME Journal of Lubrication Technology, 1975.

R. Lueptow. 2009. Schematics of rotating vortices. s.1. : Scholarpedia, 2009.

Siemens. 2020. https://www.plm.automation.siemens.com/global/en/products/simcenter/STAR-CCM.html. 2020. 